Research Article

\title{
Decision-Making Based on q-Rung Orthopair Fuzzy Soft Rough Sets
}

\author{
Yinyu Wang $\mathbb{D}^{1},{ }^{1}$ Azmat Hussain $\left(\mathbb{D},{ }^{2}\right.$ Tahir Mahmood $\mathbb{D D}^{2}$ Muhammad Irfan Ali ${ }^{(D)}{ }^{3}$ \\ Hecheng Wu, ${ }^{1}$ and Yun Jin ${ }^{4}{ }^{4}$ \\ ${ }^{1}$ College of Economic and Management, Nanjing University of Aeronautics and Astronautics, Nanjing 211106, China \\ ${ }^{2}$ Department of Mathematics and Statistics, Faculty of Basic and Applied Sciences, International Islamic University Islamabad, \\ Islamabad, Pakistan \\ ${ }^{3}$ Department of Mathematics, Islamabad Model College for Boys, G-11/1, Islamabad, Pakistan \\ ${ }^{4}$ Wuxi Vocational College of Science and Technology, Wuxi 214028, China \\ Correspondence should be addressed to Tahir Mahmood; tahirbakhat@iiu.edu.pk
}

Received 17 October 2020; Revised 11 November 2020; Accepted 23 November 2020; Published 22 December 2020

Academic Editor: Chonghui Zhang

Copyright ( 2020 Yinyu Wang et al. This is an open access article distributed under the Creative Commons Attribution License, which permits unrestricted use, distribution, and reproduction in any medium, provided the original work is properly cited.

\begin{abstract}
Recently, Yager presented the new concept of q-rung orthopair fuzzy (q-ROF) set (q-ROFS) which emerged as the most significant generalization of Pythagorean fuzzy set (PFS). From the analysis of q-ROFS, it is clear that the rung q is the most significant feature of this notion. When the rung $\mathrm{q}$ increases, the orthopair adjusts in the boundary range which is needed. Thus, the input range of q-ROFS is more flexible, resilient, and suitable than the intuitionistic fuzzy set (IFS) and PFS. The aim of this manuscript is to investigate the hybrid concept of soft set $\left(S_{t} S\right)$ and rough set with the notion of q-ROFS to obtain the new notion of q-ROF soft rough $\left(\mathrm{q}-\mathrm{ROFS}_{t} \mathrm{R}\right)$ set $\left(\mathrm{q}-\mathrm{ROFS}_{t} \mathrm{RS}\right)$. In addition, some averaging aggregation operators such as $\mathrm{q}-\mathrm{ROFS}_{t} \mathrm{R}$ weighted averaging (qROFS $_{t}$ RWA), q-ROFS R $_{t}$ ordered weighted averaging (q-ROFS $\mathrm{ROWA}_{t}$, and q-ROFS ${ }_{t} \mathrm{R}$ hybrid averaging (q-ROFS $\mathrm{RHA}_{t}$ operators are presented. Then, the basic desirable properties of these investigated averaging operators are discussed in detail. Moreover, we investigated the geometric aggregation operators, such as $\mathrm{q}-\mathrm{ROFS}_{t} \mathrm{R}$ weighted geometric (q-ROFS $\mathrm{RWG}_{t}$ ), $\mathrm{q}-\mathrm{ROFS}_{t} \mathrm{R}$ ordered weighted geometric (q-ROFS $\mathrm{ROWG}_{t}$, and $\mathrm{q}-\mathrm{ROFS}_{t} \mathrm{R}$ hybrid geometric (q-ROFS $\mathrm{RHG}^{\mathrm{R}}$ ) operators, and proposed the basic desirable characteristics of the investigated geometric operators. The technique for multicriteria decisionmaking $(\mathrm{MCDM})$ and the stepwise algorithm for decision-making by utilizing the proposed approaches are demonstrated clearly. Finally, a numerical example for the developed approach is presented and a comparative study of the investigated models with some existing methods is brought to light in detail which shows that the initiated models are more effective and useful than the existing methodologies.
\end{abstract}

\section{Introduction}

Decision-making has always been a hot topic under consideration by the researchers. Multicriteria decision-making (MCDM) has a high prospective and discipline manner to improve and evaluate multiple conflicting criteria in all areas of decision-making. In this competitive environment, an enterprise needs the most accurate and rapid response to change the customer needs. So, MCDM has the ability to handle successfully the evaluation process of multiple contradictory criteria. For an intelligent decision, the experts analyze each and every character of an alternative and then they take the decision. For an intelligent and successful decision, the experts require careful preparation and analysis of each and every character for an alternative and then they can take a good decision if they are armed with all the data and information that they need. To handle this complexity, Zadeh [1] originated the dominant and pioneer concept of the fuzzy set. For each domain in the fuzzy set, a value is assigned from the unit interval and is called membership grade (MemG). From the inception of the fuzzy set, it has been generalized in different directions in which one of the 
most significant concepts is intuitionistic fuzzy (IF) set (IFS). Atanassov [2] initiated this dominant concept of IFS which is characterized by two mappings called MemG and nonmembership grade (NMemG). IFS is defined on the basis of restriction that the sum of MemG and NMemG must not exceed the unit interval $[0,1]$. The notion of IFS appears as a hot research area after its origination. In literature, researchers used different techniques to handle the ranking with score or accuracy functions but all these techniques had some drawbacks. So, Ali et al. [3] initiated a graphical technique for ranking the IF values. Xu [4] investigated the series of aggregation operators such as IF weighted averaging (IFWA), IF ordered weighted averaging (IFOWA), and IF hybrid averaging (IFHA) operators under IF environment. The series of geometric operators, namely, IF weighted geometric (IFWG), IF ordered weighted geometric (IFOWG), and IF hybrid geometric (IFHG) operators, were presented by $\mathrm{Xu}$ and Yager [5]. Zhao et al. [6] initiated the idea of generalized IFWA, generalized IFOWA, and generalized IFHA operators by utilizing the IF information. Wang and Liu $[7,8]$ originated the concepts of geometric and averaging aggregation operators by utilizing Einstein operations. Zeng et al. [9] investigated a novel score function of intuitionistic fuzzy and then presented its application in decision-making. In a different scenario, the professional experts are restricted to provide their choices in the range of IFS. To cover this shortcoming, Yager [10] investigated the powerful paradigm of Pythagorean fuzzy (PF) set (PFS) in which the square sum of MemG and NMemG must lie between the real numbers 0 and 1 . PFS relaxes and widens the boundary range by providing additional space to the decision-maker. Yager [11] originated the geometric and averaging aggregation operation by using PF information. Peng and Yang [12] initiated the concept of subtraction and division operators and proved some of its basic properties. Peng and Yang [13] investigated the notion of PF Choquet integral average and PF Choquet integral geometric operators. Garg $[14,15]$ proposed some PF Einstein averaging and PF Einstein geometric operators and presented their basic characteristics. Garg [16] investigated confidence PF weighted and ordered weighted averaging operators with their basic properties. Wei and $\mathrm{Lu}$ [17] originated the notions of PF power averaging and geometric operators and presented their desirable characteristics of these investigated operators. Wei [18] presented some interaction averaging and geometric operators by suing PF information. The concept of Hamacher operations for PF averaging and geometric operators was presented by $\mathrm{Wu}$ and Wei [19]. In the PF environment, the decision-makers are restricted to their boundary limitation and they cannot provide their preferred values freely. Due to these restrictions, some decisive information cannot be effectively handled by PFS.

Recently, Yager [20] presented the new concept of q-rung orthopair fuzzy (q-ROF) set (q-ROFS) from which the most significant generalization of PFS emerged. In q-ROFS, the sum of $q$ th power of MemG and $q$ th power of NMemG must be confined to the unit interval $[0,1]$ and, furthermore, when the rung $q$ increases, then the range of orthopair satisfies the boundary restriction which is needed.
Thus, the concept of q-ROFS is more useful and powerful than IFS and PFS because these are the special cases of q-ROFS. The basic properties of q-ROFS are proposed by Yager and Alajlan [21] and have been utilized in knowledge representation. Ali [22] proposed another view of q-ROFS by using the idea of orbits. Liu and Wang [23] proposed the concepts of q-ROF weighted averaging (q-ROFWA) and q-ROF weighted geometric (q-ROFWG). Liu and Liu [24] presented the combined study of Bonferroni mean (BM) operators with q-ROFS to investigate the q-ROF BM operators and also study q-ROF geometric BM operators with their desirable properties. Jana et al. [25] initiated the q-ROF Dombi averaging and geometric aggregation operators with their fundamental desirable characteristics. Wang et al. [26] investigated the combined concept of Muirhead means (MM) operators with q-ROFS to get the new aggregation operators that are q-ROF MM operators. Joshi and Gegov [27] initiated the concept of the confidence level of experts to the original information under q-ROF environment to propose some aggregation operators such as confidence q-ROFWA (Cq-ROFWA) and confidence q-ROFWG (CqROFWG), Cq-ROFOWA, and Cq-ROFOWG operators. Yang et al. [28] presented the idea of q-RO normal fuzzy sets and defined the operational laws and score function for it. They also initiated some aggregation operators for the same concept that is q-RONFWA and q-RONFOWG. Furthermore, Hussain et al. [29] proposed hesitant q-ROFWA and hesitant q-ROFWG operators and discussed their desirable properties. Hussain et al. [30] proposed the generalized and group generalized averaging operation by using q-ROF information.

The dominant theory of rough set was first proposed by Pawlak [31] which generalized the classical set theory to cope with the imprecise, vague, and uncertain information. By the definition of Pawlak rough set, a universal set is characterized by two approximation sets known as upper and lower approximations. The lower approximation consists of those alternatives which contain a subset and the upper approximation consists of those alternatives having nonempty intersection with a subset. Further equivalence relation plays a key role in Pawlak rough set for approximations but this condition too restricts the practical and theoretical aspects of rough set. So, researchers used the generalized structure by using the nonequivalence structure; for details, see [32-38]. From the inception, researchers used the hybrid study of rough set theory with different concepts. The hybrid study of rough set and IFS was proposed by Chakrabarty et al. [39] to obtain the notion of IF rough set (IFRS) and IFRS became the hot and progressive research area for the researchers; for details, see [40-43]. Zhou and $\mathrm{Wu}$ [44] proposed the combined study of rough set and IFS by using crisp and fuzzy approximation. Zhou and $\mathrm{Wu}$ [45] initiated the constructive and axiomatic approach under the IF rough environment. Hussain et al. [46] investigated the idea of rough PF ideals by using the algebraic structure of semigroups. Zeng [47] proposed a new MCDM technique based on probabilistic information by using the $\mathrm{PF}$ environment. Hussain et al. [48] proposed the notion of q-ROF rough set by utilizing fuzzy $\beta$-covering and fuzzy $\beta$-covering 
neighborhood. Molodtsov [49] originated the prominent and pioneer concept of soft set $\left(S_{t} S\right)$ which generalized the classical set theory and is free from inherent complexity which the contemporary theories faced. It is observed that $S_{t} S$ has a very close relation with fuzzy set and rough set. The $S_{t} S$ theory is an essential concept and powerful mathematical tool for coping the uncertain, ambiguous, and imprecise data. Maji et al. [50,51] proposed the hybrid notion of $S_{t} S$ with fuzzy set and IFS to obtain fuzzy $S_{t} S$ and IFS $S$ which play a key role among these theories. Ali et al. [52] improved some existing definitions and operations in $S_{t} S$ theory. The concept of generalized IFS $S_{t}$ was proposed by Agarwal et al. [53]. Arora and Garg [54] presented the concept of IFS weighted averaging $\left(\right.$ IFS $\left._{t} \mathrm{WA}\right)$ and IFS seighted geometric $_{t}$ $\left(\mathrm{IFS}_{t} \mathrm{WG}\right.$ ) operators. Garg and Arora [55] proposed the notion of some power averaging and geometric aggregation operators by utilizing generalized IF $S_{t} S$. Arora [56] initiated the notion of IFS ${ }_{t} \mathrm{WA}$ and IFS $S_{t} \mathrm{WG}$ by using the Einstein operations. Feng et al. [57] improved some existing literature related to generalized IF $S_{t} S$ and proposed some new operations for the developed concept. The combined study $S_{t} S$, rough set, and PFS were presented by Hussain et al. [58] to achieve the new concept of soft rough PFS and PF soft rough set. Riaz and Hashmi [59] presented the hybrid study of $S_{t} S$, rough set, PFS, and $\mathrm{m}$-polar fuzzy set to get the new notion of Pythagorean m-polar fuzzy soft rough set. Hussain et al. [60] originated the hybrid structure of $S_{t} S$ with q-ROFS to get the prominent notion of q-ROF soft (q-ROFS ${ }_{t}$ ) set (q$\mathrm{ROFS}_{t} \mathrm{~S}$ ) and proposed some aggregation operators such as $\mathrm{q}-\mathrm{ROFS}_{t}$ weighted averaging (q-ROFS $\left.t \mathrm{WA}\right), \mathrm{q}-\mathrm{ROFS}_{t}$ ordered weighted averaging (q-ROFS $S_{t} \mathrm{OWA}$ ), and $\mathrm{q}-\mathrm{ROFS}_{t}$ hybrid averaging (q-ROFS $\mathrm{HA}$ ).

q-Rung orthopair fuzzy soft rough sets, a hybrid intelligent structure of soft sets, rough sets, and q-rung orthopair fuzzy sets are a powerful mathematical tool to deal with indeterminate, inconsistent, and incomplete information, which has caught the attention of the researchers. From the analysis, it is observed that aggregation operators have great importance in decision-making to aggregate the collective evaluated information of different sources into a single value. According to the best of our knowledge up till now, no application of the aggregation operators with the hybridization of q-ROFS with soft set and rough set is reported in q-ROF environment. Therefore, this motivates the current work of q-ROFS $S_{t}$ rough study, and, further, we will investigate aggregation operators based on soft rough information that are $\mathrm{q}-\mathrm{ROFS}_{t} \mathrm{RWA}^{\mathrm{R}} \mathrm{q}-\mathrm{ROFS}_{t} \mathrm{ROWA} \mathrm{q}-\mathrm{ROFS}_{t} \mathrm{RHA}$, $\mathrm{q}-\mathrm{ROFS}_{t} \mathrm{RWG}, \mathrm{q}-\mathrm{ROFS}_{t}$ ROWG, and $\mathrm{q}-\mathrm{ROFS}_{t}$ RHG operators.

The design of the remaining sections of the manuscript is summarized as follows: Section 2 consists of a brief study of the basic notions connecting the link with the coming sections. Section 3 is devoted to investigating the hybrid concept of $S_{t} S$ and rough set with the notion of q-ROFS to obtain the new concept of q-ROFS ${ }_{t}$ RS. In Section 4, we presented the averaging aggregation operators such as q-ROFS $S_{t}$ RWA, q-ROFS ${ }_{t}$ ROWA, and q-ROFS ${ }_{t}$ RHA. Furthermore, the basic desirable properties of investigated averaging operators that are Idempotency, Boundedness,
Monotonicity, shift invariance, and Homogeneity are investigated in detail. Section 5 is devoted to the geometric aggregation operators such as q-ROFS ${ }_{t}$ RWG, $\mathrm{q}-\mathrm{ROFS}_{t}$ ROWG, and q-ROFS ${ }_{t}$ RHG. Moreover, the basic desirable characteristics of these investigated geometric operators that are Idempotency, Boundedness, Monotonicity, shift invariance, and Homogeneity are investigated in detail. In Section 6, the technique for MCDM and the stepwise algorithm for decision-making are demonstrated by utilizing the proposed approach. In Section 7, a numerical example for the developed approach is presented and a comparative study of the investigated models with some existing methods is given in detail which shows that the investigated models are more effective and useful than existing approaches. The final Section 8 consists of a conclusion of the manuscript.

\section{Preliminaries}

This section consists of some basic notions including IFS, PFS, q-ROFS, and $\mathrm{q}-\mathrm{ROFS}_{t} \mathrm{~S}$ which will be helpful in on word sections.

Definition 1 (see [2]). Consider a universe $Y$ and an IFST on set $Y$ denoted and defined as

$$
\mathscr{T}=\left\{\left\langle s, \beta_{\mathscr{T}}(s), \vartheta_{\mathscr{T}}(s)\right\rangle \mid s \in Y\right\},
$$

in which $\beta_{\mathscr{T}}, \vartheta_{\mathscr{T}}: Y \longrightarrow[0,1]$ denotes the MemG and NMemG of an alternative $s \in Y$ to the set $\mathscr{T}$ having the condition that $0 \leq \beta_{\mathscr{T}}(s)+\vartheta_{\mathscr{T}}(s) \leq 1 . \pi_{\mathscr{T}}=1-\left(\beta_{\mathscr{T}}(s)+\right.$ $\left.\vartheta_{\mathscr{T}}(s)\right)$ is called hesitancy degree of $s \in Y$.

Definition 2 (see [10]). Consider a universe $Y$ and a PFST on set $Y$ defined and denoted as

$$
\mathscr{T}=\left\{\left\langle s, \beta_{\mathscr{T}}(s), \vartheta_{\mathscr{T}}(s)\right\rangle \mid s \in Y\right\},
$$

in which $\beta_{\mathscr{T}}, \vartheta_{\mathscr{T}}: Y \longrightarrow[0,1]$ denotes the MemG and NMemG of an alternative $s \in Y$ to the set $\mathscr{T}$ having the condition that $0 \leq\left(\beta_{\mathscr{T}}(s)\right)^{2}+\left(\vartheta_{\mathscr{T}}(s)\right)^{2} \leq 1$. $\pi_{\mathscr{T}}=\sqrt{1-\left(\beta_{\mathscr{T}}(s)\right)^{2}-\left(\vartheta_{\mathscr{T}}(s)\right)^{2}}$ is called hesitancy degree of $s \in Y$.

Definition 3 (see [20]). Consider $Y$ as a universe of discourse and a $\mathrm{q}-\mathrm{ROFS} \mathscr{T}$ on set $Y$ is an object of the form

$$
\mathscr{T}=\left\{\left\langle s, \beta_{\mathscr{T}}(s), \vartheta_{\mathscr{T}}(s)\right\rangle_{q} \mid s \in Y \text { and } q \geq 1\right\},
$$

in which $\beta_{\mathscr{T}}, \vartheta_{\mathscr{T}}: Y \longrightarrow[0,1]$ shows the MemG and NMemG of an alternative $s \in Y$ to the set $\mathscr{T}$ having the condition that $0 \leq\left(\beta_{\mathscr{T}}(s)\right)^{q}+\left(\vartheta_{\mathscr{T}}(s)\right)^{q} \leq 1$. The hesitancy degree is shown as $\pi_{\mathscr{T}}=\sqrt[q]{1-\left(\beta_{\mathscr{T}}(s)\right)^{q}-\left(\vartheta_{\mathscr{T}}(s)\right)^{q}}$ for each $s \in Y$.

Molodtsov [49] originated the prominent and pioneer concept of soft set $\left(S_{t} S\right)$ which generalized the classical set theory and is free from inherent complexity which the contemporary theories faced. It is observed that $S_{t} S$ has very close relation with fuzzy set and rough set. The $S_{t} S$ theory is an essential concept and powerful mathematical tool for 
coping the uncertain, ambiguous and imprecise data which is defined as:

Definition 4 (see [49]). Let $Y$ be a fixed set and $\mathscr{E}$ be set of parameters with $\mathscr{B} \subseteq \mathscr{E}$. Then the pair $(\mathscr{T}, \mathscr{B})$ is said to be $S_{t} S$, where $\mathscr{T}$ is function given as $\mathscr{T}: \mathscr{B} \longrightarrow P(Y) . P(Y)$ denotes the collection of all subsets of $Y$.

Definition 5 (see [50]). Let $(\mathscr{T}, \mathscr{B})$ a $S_{t} S$ over $Y$ with $\mathscr{B} \subseteq \mathscr{E}$. Then $\left(\mathscr{T}^{*}, \mathscr{B}\right)$ is known as fuzzy $S_{t} S$ over $Y$, where $\mathscr{T}^{*}$ is a function given as $\mathscr{T}^{*}: \mathscr{B} \longrightarrow F(Y)$. $F(Y)$ denotes the collection of all fuzzy subsets of $Y$ and mathematically it is gives as

$$
\mathscr{T}_{c_{j}}^{*}=\left\{\left\langle s_{i}, \beta_{j}\left(s_{i}\right)\right\rangle \mid s_{i} \in Y\right\} .
$$

Definition 6 (see [60]). Consider a universal set $Y$. Let $\mathscr{E}$ be set of parameters and $\mathscr{B} \subseteq \mathscr{E}$. Then a q-ROFS $t$ S is a pair $(\mathscr{T}, \mathscr{B})$ over set $Y$ and $\mathscr{T}$ is a mapping given as $\mathscr{T}: \mathscr{B} \longrightarrow \mathrm{q}-\mathrm{ROFS}_{t} \mathrm{~S}(Y)$ in which $\mathrm{q}-\mathrm{ROFS}_{t} \mathrm{~S}(Y)$ contains the collection of all q-ROFSs. Then, the q-ROFS $t$ S is denoted and defined as

$$
\mathscr{T}_{c_{j}}\left(s_{i}\right)=\left\{\left\langle s_{i}, \beta_{j}\left(s_{i}\right), \vartheta_{j}\left(s_{i}\right)\right\rangle_{q} \mid s_{i} \in Y \text { and } q \geq 1\right\},
$$

in which $\beta_{j}\left(s_{i}\right), \vartheta_{j}\left(s_{i}\right)$ represents the MemG and NMemG of an alternative $s_{i} \in Y$ to the set $\mathscr{T}_{c_{j}}$ satisfying that $0 \leq\left(\beta_{j}\left(s_{i}\right)\right)^{q}+\left(\vartheta_{j}\left(s_{i}\right)\right)^{q} \leq 1$.

$\pi_{\mathscr{T}}=\sqrt[q]{1-\left(\beta_{j}\left(s_{i}\right)\right)^{q}-\left(\vartheta_{j}\left(s_{i}\right)\right)^{q}}$ is known to be hesitancy degree of $s_{i} \in Y$. For simplicity $\mathscr{T}_{c_{j}}\left(s_{i}\right)=\left(s_{i}, \beta_{j}\left(s_{i}\right)\right.$, $\left.\vartheta_{j}\left(s_{i}\right)_{q}\right)$ is denoted as $\mathscr{T}_{c_{j}}=\left(\beta_{i j}, \vartheta_{i j}\right)$ if there is no confusion and is called q-ROFS $S_{t}$ number $\left(\mathrm{q}-\mathrm{ROFS}_{t} \mathrm{~N}\right)$.

Considering two q-ROFS $\mathrm{Ns} \mathscr{T}_{c_{j}}=\left(\beta_{1 j}, \vartheta_{1 j}\right)$ for $(j=1,2)$, reference [20] defined the following operation are defined on them:

$$
\text { (i) } \mathscr{T}_{c_{1}} \cup \mathscr{T}_{c_{2}}=\left(\max \left(\beta_{11}, \beta_{12}\right), \min \left(\vartheta_{11}, \vartheta_{12}\right)\right)
$$

(ii) $\mathscr{T}_{c_{1}} \cap \mathscr{T}_{c_{2}}=\left(\min \left(\beta_{11}, \beta_{12}\right), \max \left(\vartheta_{11}, \vartheta_{12}\right)\right)$

(iii) $\mathscr{T}_{c_{1}} \oplus \mathscr{T}_{c_{2}}=\left(\sqrt[q]{\beta_{11}^{q}+\beta_{12}^{q}-\beta_{11}^{q} \beta_{12}^{q}}, \vartheta_{11} \vartheta_{12}\right)$

(iv) $\mathscr{T}_{c_{1}} \oplus \mathscr{T}_{c_{2}}=\left(\beta_{11} \beta_{12}, \sqrt[q]{\vartheta_{11}^{q}+\vartheta_{12}^{q}-\vartheta_{11}^{q} \vartheta_{12}^{q}}\right)$

(v) $\mathscr{T}_{c_{1}} \leq \mathscr{T}_{c_{2}}$ if $\left(\beta_{11} \leq \beta_{12}, \vartheta_{11} \geq \vartheta_{12}\right)$

(vi) $\mathscr{T}_{c_{1}}^{c}=\left(\vartheta_{11}, \beta_{11}\right)$ where $\mathscr{T}_{c_{1}}^{c}$ represents the complement of $\mathscr{T}_{c_{1}}$

(vii) $\alpha \mathscr{T}_{c_{1}}=\left(\sqrt[q]{1-\left(1-\beta_{11}^{q}\right)^{\alpha}}, \vartheta_{11}^{\alpha}\right)$ for $\alpha \geq 1$

(viii) $\mathscr{T}_{c_{1}}^{\alpha}=\left(\beta_{11}^{\alpha}, \sqrt[q]{1-\left(1-\vartheta_{11}^{q}\right)^{\alpha}}\right)$ for $\alpha \geq 1$

\section{3. q-ROF Soft Rough Set (q-ROF $S_{t}$ RS)}

The concept of $S_{t} S$ theory, is the generalization of classical set theory and is free from inherent complexity which the contemporary theories faced. The $S_{t} S$ theory and rough set theory are essential concepts and powerful mathematical tools for coping the uncertain, ambiguous, and imprecise data. Motivated from the combining study of soft rough set, this section is devoted to the hybridized study of q-ROFS with $S_{t} S$ and rough set to obtain the new concept of
$\mathrm{q}-\mathrm{ROFS}_{t} \mathrm{RS}$. Some basic operations, a new score function, and some desirable characteristics of the proposed concept are investigated in detail.

Definition 7. Let $(\mathscr{T}, \mathscr{E})$ be a $\mathrm{q}-\mathrm{ROFS}_{t} \mathrm{~S}$ over $Y$. Any subset

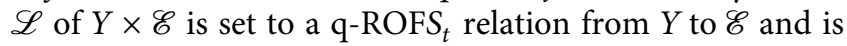
defined as

$$
\mathscr{L}=\left\{\left\langle\left(s_{i}, c_{j}\right), \beta\left(s_{i}, c_{j}\right), \vartheta\left(s_{i}, c_{j}\right)\right\rangle \mid\left(s_{i}, c_{j}\right) \in Y \times \mathscr{E}\right\},
$$

where $\beta: Y \times \mathscr{E} \longrightarrow[0,1]$ and $\vartheta: Y \times \mathscr{E} \longrightarrow[0,1]$ denote the MemG and NMemG with $0 \leq\left[\beta\left(s_{i}, c_{j}\right)\right]^{q}+\left[\vartheta\left(s_{i}, c_{j}\right)\right]^{q} \leq 1$ for all $\left(s_{i}, c_{j}\right) \in Y \times \mathscr{E}$.

If $Y=\left\{s_{1}, s_{2}, \ldots, s_{m}\right\}$ and $\mathscr{E}=\left\{c_{1}, c_{2}, \ldots, c_{n}\right\}$, then, q-ROFS $S_{t}$ relation $\mathscr{L}$ from $Y$ to $\mathscr{E}$ can be presented in Table 1.

Definition 8. Consider a universal $Y, \mathscr{E}$ as the set of parameters and $(\mathscr{T}, \mathscr{E})$ as a $\mathrm{q}-\mathrm{ROFS}_{t} \mathrm{~S}$. Let $\mathscr{L}$ be an arbitrary q-ROFS $S_{t}$ relation from set $Y$ to $\mathscr{E}$. The pair $(\mathscr{T}, \mathscr{E}, \mathscr{L})$ is said

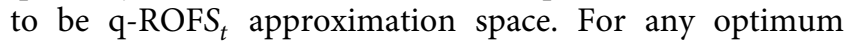
decision normal object $\mathscr{M} \in \mathrm{q}-\operatorname{ROFS}(\mathscr{E})$, then the lower and upper approximation of $\mathscr{M}$ with respect to approximation space $(\mathscr{T}, \mathscr{E}, \mathscr{L})$ are denoted and defined as

$$
\begin{aligned}
& \underline{\mathscr{L}}(\mathscr{M})=\left\{\left(s_{i}, \underline{\beta_{j}}\left(s_{i}\right), \underline{\vartheta_{j}}\left(s_{i}\right)\right) \mid s_{i} \in Y\right\}, \\
& \overline{\mathscr{L}}(\mathscr{M})=\left\{\left(s_{i}, \overline{\beta_{j}}\left(s_{i}\right), \overline{\vartheta_{j}}\left(s_{i}\right)\right) \mid s_{i} \in Y\right\},
\end{aligned}
$$

where $\underline{\beta_{j}}\left(s_{i}\right)=\wedge_{c_{j} \in \mathscr{E}}\left[\beta_{\mathscr{L}}\left(s_{i}, c_{j}\right) \wedge \beta_{\mathscr{M}}\left(c_{j}\right)\right], \underline{\vartheta_{j}}\left(s_{i}\right)=\vee_{c_{j} \in \mathscr{E}}\left[\vartheta_{\mathscr{L}}\right.$ $\left.\left(s_{i}, c_{j}\right) \bar{\vartheta}_{\mathscr{M}}\left(c_{j}\right)\right]$ and $\overline{\beta_{j}}\left(s_{i}\right)=\vee_{c_{j} \in \mathscr{E}}\left[\overline{\beta_{\mathscr{L}}}\left(s_{i}, c_{j}\right) \vee \beta_{\mathscr{M}}\left(c_{j}\right)\right]$, ${\overline{\vartheta_{j}}}_{j}\left(s_{i}\right)=\wedge_{c_{j} \in \mathscr{E}}\left[\vartheta_{\mathscr{L}}\left(s_{i}, c_{j}\right) \wedge \vartheta_{\mathscr{M}}\left(c_{j}\right)\right] \quad$ such that $0 \leq\left[\underline{\beta_{j}}\left(s_{i}\right)\right]^{q}+\left[\underline{\vartheta_{j}}\left(s_{i}\right)\right]^{q} \leq 1$ and $0 \leq\left[\overline{\beta_{j}}\left(s_{i}\right)\right]^{q}+\left[\overline{\vartheta_{j}}\left(s_{i}\right)\right]^{q} \leq 1$.

It is observed that $\underline{\mathscr{L}}(\mathscr{M})$ and $\overline{\mathscr{L}}(\mathscr{M})$ are two q-ROFSs in $Y$. Thus, the operators $\mathscr{L}(\mathscr{M}), \overline{\mathscr{L}}(\mathscr{M}): \mathrm{q}-\mathrm{ROFS}_{t}$ $(\mathscr{E}) \longrightarrow \mathrm{q}-\operatorname{ROFS}_{t}(Y)$ are, respectively, known as lower and upper q-ROFS $\mathrm{R}_{t}$ approximation operators. Therefore, q-ROFS ${ }_{t}$ RS is a pair $\mathscr{L}(\mathscr{M})=(\underline{\mathscr{L}}(\mathscr{M}), \overline{\mathscr{L}}$ $(\mathscr{M}))=\left(s_{i},\left(\beta_{j}\left(s_{i}\right), \underline{\vartheta_{j}}\left(s_{i}\right)\right),\left(\overline{\beta_{j}}\left(s_{i}\right), \overline{\vartheta_{j}}\left(s_{i}\right)\right)\right)$.

For simplicity, we can write $\mathscr{L}(\mathscr{M})=(\underline{\mathscr{L}}(\mathscr{M}), \overline{\mathscr{L}}(\mathscr{M}))=$ $\left(s_{i},\left(\underline{\beta_{j}}\left(s_{i}\right), \underline{\vartheta_{j}}\left(s_{i}\right)\right),\left(\overline{\beta_{j}}\left(s_{i}\right), \overline{\vartheta_{j}}\left(s_{i}\right)\right)\right) \quad$ as $\quad \mathscr{L}_{c_{j}}\left(\mathscr{M}_{i}\right)=\left(\mathscr{L}_{c_{j}}\right.$ $\left.\left(\mathscr{M}_{i}\right), \overline{\mathscr{L}_{c_{j}}}\left(\overline{\mathscr{M}_{i}}\right)\right)=\left(\left(\beta_{i j}, \vartheta_{i j}\right)\right),\left(\overline{\beta_{i j}}, \overline{\vartheta_{i j}}\right)$ and call q-ROFS $\mathrm{R}$ number $\left(\mathrm{q}-\mathrm{ROFS}_{t} \mathrm{RN}\right)$, if there is no confusion.

Remark 1

(a) If $q=1$ is fixed, then the developed $q-\mathrm{ROFS}_{t} \mathrm{R}$ approximation operators reduce to $\mathrm{IFS}_{t} \mathrm{R}$ approximation operators

(b) If $q=2$ is fixed, then the developed $\mathrm{q}-\mathrm{ROFS}_{t} \mathrm{R}$ approximation operators reduce to $\mathrm{PFS}_{t} \mathrm{R}$ approximation operators

Consider the following example to better understand the concept of $\mathrm{q}-\mathrm{ROFS}_{t} \mathrm{R}$ approximation operators.

Example 1. Suppose a decision-maker $Z$ buys a house, as given in set $Y=\left\{s_{1}, s_{2}, s_{3}, s_{4}, s_{5}\right\}$ under consideration. Let the 
TABLe 1: q-ROFS ${ }_{t}$ relation $\mathscr{L}$ from $Y$ to $\mathscr{E}$.

\begin{tabular}{ccccc}
\hline $\mathscr{L}$ & $c_{1}$ & $c_{2}$ & $\cdots$ & $c_{n}$ \\
\hline$s_{1}$ & $\left(\beta\left(s_{1}, c_{1}\right), \vartheta\left(s_{1}, c_{1}\right)\right)$ & $\left(\beta\left(s_{1}, c_{2}\right), \vartheta\left(s_{1}, c_{2}\right)\right)$ & $\ldots$ & $\left(\beta\left(s_{1}, c_{n}\right), \vartheta\left(s_{1}, c_{n}\right)\right)$ \\
$s_{2}$ & $\left(\beta\left(s_{2}, c_{1}\right), \vartheta\left(s_{2}, c_{1}\right)\right)$ & $\left(\beta\left(s_{2}, c_{2}\right), \vartheta\left(s_{2}, c_{2}\right)\right)$ & $\vdots$ & $\left(\beta\left(s_{2}, c_{n}\right), \vartheta\left(s_{2}, c_{n}\right)\right)$ \\
$\vdots$ & $\vdots$ & $\vdots$ & $\ddots$ & $\vdots$ \\
$s_{m}$ & $\left(\beta\left(s_{m}, c_{1}\right), \vartheta\left(s_{m}, c_{1}\right)\right)$ & $\left(\beta\left(s_{m}, c_{2}\right), \vartheta\left(s_{m}, c_{2}\right)\right)$ & $\ldots$ & $\left(\beta\left(s_{m}, c_{n}\right), \vartheta\left(s_{m}, c_{n}\right)\right)$ \\
\hline
\end{tabular}

parameter set $\mathscr{E}=\left\{c_{1}, c_{2}, c_{3}, c_{4}\right\}$ where $c_{1}=$ beautiful, $c_{2}=$ size, $c_{3}=$ expensive, and $c_{4}=$ location. A decisionmaker $Z$ wants to purchase a house from the available houses which fulfill the utmost extent of given parameters. Consider the decision-maker $Z$ presents the gorgeous of houses in form of q-ROFS $S_{t}$ relation $\mathscr{L}$ from set $Y$ to $\mathscr{E}$ and is given in Table 2.

Consider a decision-maker $Z$ presents the optimum normal decision object $\mathscr{M}$ which is a q-ROF subset over parameter set $\mathscr{E}$; that is,

$\mathscr{M}=\left\{\left(c_{1}, 0.9,0.2\right),\left(c_{2}, 0.4,0.6\right),\left(c_{3}, 0.8,0.4\right),\left(c_{4}, 0.5,0.1\right)\right\}$.

Now, by using equations (7) and (8), we have

$$
\begin{aligned}
& \underline{\beta_{1}}\left(s_{1}\right)=0.4, \\
& \underline{\vartheta_{1}}\left(s_{1}\right)=0.6, \\
& \underline{\beta_{2}}\left(s_{2}\right)=0.3, \\
& \underline{\vartheta_{2}}\left(s_{2}\right)=0.7, \\
& \underline{\beta_{3}}\left(s_{3}\right)=0.2, \\
& \overline{\vartheta_{3}}\left(s_{3}\right)=0.9, \\
& \overline{\beta_{4}}\left(s_{4}\right)=0.4, \\
& \overline{\vartheta_{4}}\left(s_{4}\right)=0.6, \\
& \overline{\beta_{5}}\left(s_{5}\right)=0.3, \\
& \overline{\vartheta_{5}}\left(s_{5}\right)=0.7, \\
& \overline{\beta_{1}}\left(s_{1}\right)=0.9, \\
& \overline{\vartheta_{1}}\left(s_{1}\right)=0.1, \\
& \overline{\beta_{2}}\left(s_{2}\right)=0.9, \\
& \overline{\vartheta_{2}}\left(s_{2}\right)=0.1, \\
& \overline{\beta_{3}}\left(s_{3}\right)=0.95, \\
& \overline{\vartheta_{3}}\left(s_{3}\right)=0.1 \\
& \overline{\beta_{4}}\left(s_{4}\right)=0.93, \\
& \overline{\vartheta_{4}}\left(s_{4}\right)=0.1, \\
& \overline{\beta_{5}}\left(s_{5}\right)=0.9,
\end{aligned}
$$

Now, to get the lower and upper q-ROFS $\mathrm{R}_{t}$ approximation operators,
TABLE 2: q-ROFS $S_{t}$ relation from set $Y$ to $\mathscr{E}$ for $q \geq 3$.

\begin{tabular}{ccccc}
\hline $\mathscr{L}$ & $c_{1}$ & $c_{2}$ & $c_{3}$ & $c_{4}$ \\
\hline$s_{1}$ & $(0.9,0.4)$ & $(0.8,0.2)$ & $(0.7,0.3)$ & $(0.65,0.2)$ \\
$s_{2}$ & $(0.8,0.5)$ & $(0.5,0.1)$ & $(0.85,0.2)$ & $(0.3,0.7)$ \\
$s_{3}$ & $(0.6,0.9)$ & $(0.2,0.6)$ & $(0.6,0.1)$ & $(0.95,0.3)$ \\
$s_{4}$ & $(0.7,0.4)$ & $(0.93,0.4)$ & $(0.4,0.2)$ & $(0.5,0.1)$ \\
$s_{5}$ & $(0.3,0.7)$ & $(0.78,0.25)$ & $(0.8,0.15)$ & $(0.7,0.4)$ \\
\hline
\end{tabular}

$$
\begin{aligned}
\mathscr{L}(\mathscr{M})= & \left\{\left(s_{1}, 0.7,0.6\right),\left(s_{2}, 0.3,0.7\right),\left(s_{3}, 0.2,0.9\right),\right. \\
& \left.\left(s_{4}, 0.4,0.6\right),\left(s_{5}, 0.3,0.7\right)\right\}, \\
\overline{\mathscr{L}}(\mathscr{M})= & \left\{\left(s_{1}, 0.9,0.1\right),\left(s_{2}, 0.9,0.1\right),\left(s_{3}, 0.95,0.1\right),\right. \\
& \left.\left(s_{4}, 0.93,0.1\right),\left(s_{5}, 0.9,0.1\right)\right\} .
\end{aligned}
$$

Therefore,

$$
\begin{aligned}
\mathscr{L}(\mathscr{M})= & (\mathscr{L}(\mathscr{M}), \overline{\mathscr{L}}(\mathscr{M}))=\left\{\left(s_{1},(0.7,0.6),(0.9,0.1)\right)\right. \\
& \left(s_{2},(0.3,0.7),(0.9,0.1)\right),\left(s_{3},(0.2,0.9)\right. \\
& (0.95,0.1)),\left(s_{4},(0.4,0.6),(0.93,0.1)\right) \\
& \left.\left(s_{5},(0.3,0.7),(0.9,0.1)\right)\right\}
\end{aligned}
$$

Definition 9. Consider $\mathscr{L}_{c_{j}}\left(\mathscr{M}_{1}\right)=\left(\mathscr{L}_{c_{j}}\left(\mathscr{M}_{1}\right), \overline{\mathscr{L}_{c_{j}}}\left(\mathscr{M}_{1}\right)\right)$

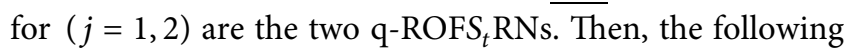
operators are defined on them:

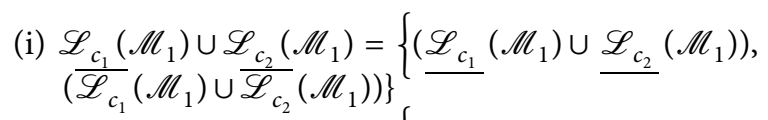

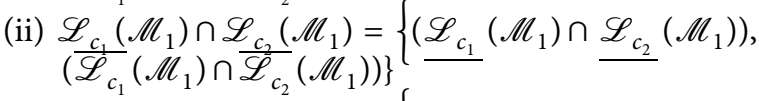

(iii) $\mathscr{L}_{c_{1}}\left(\mathscr{M}_{1}\right) \oplus \mathscr{L}_{c_{2}}\left(\mathscr{M}_{1}\right)=\left\{\left(\mathscr{L}_{c_{1}}\left(\mathscr{M}_{1}\right) \oplus \underline{\mathscr{L}_{c_{2}}}\left(\mathscr{M}_{1}\right)\right)\right.$, $\left.\left(\overline{\mathscr{L}_{c_{1}}}\left(\mathscr{M}_{1}\right) \oplus \overline{\mathscr{L}_{c_{2}}}\left(\mathscr{M}_{1}\right)\right)\right\}$

(iv) $\mathscr{L}_{c_{1}}\left(\mathscr{M}_{1}\right) \otimes \mathscr{L}_{c_{2}}\left(\mathscr{M}_{1}\right)=\left\{\left(\mathscr{L}_{c_{1}}\left(\mathscr{M}_{1}\right) \otimes \mathscr{L}_{c_{2}}\left(\mathscr{M}_{1}\right)\right)\right.$, $\left.\left(\overline{\mathscr{L}_{c_{1}}}\left(\mathscr{M}_{1}\right) \otimes \overline{\mathscr{L}_{c_{2}}}\left(\mathscr{M}_{1}\right)\right)\right\}$

(v) $\mathscr{L}_{c_{1}}\left(\mathscr{M}_{1}\right) \subseteq \mathscr{L}_{c_{2}}\left(\mathscr{M}_{1}\right)=\left(\mathscr{L}_{c_{1}}\left(\mathscr{M}_{1}\right) \subseteq \mathscr{L}_{c_{2}}\left(\mathscr{M}_{1}\right)\right)$ and $\left(\overline{\mathscr{L}_{c_{1}}}\left(\mathscr{M}_{1}\right) \subseteq \overline{\mathscr{L}_{c_{2}}}\left(\mathscr{M}_{1}\right)\right)$

(vi) $\alpha \mathscr{L}_{c_{1}}\left(\mathscr{M}_{1}\right)=\left(\alpha \mathscr{L}_{c_{1}}\left(\mathscr{M}_{1}\right), \alpha \overline{\mathscr{L}_{c_{1}}}\left(\mathscr{M}_{1}\right)\right)$ for $\alpha \geq 1$

(vii) $\left.\left(\mathscr{L}_{c_{1}}\left(\mathscr{M}_{1}\right)\right)^{\alpha}=\left(\overline{\left(\overline{\mathscr{L}_{c_{1}}}\right.}\left(\mathscr{M}_{1}\right)\right)^{\alpha},\left(\overline{\mathscr{L}_{c_{1}}}\left(\mathscr{M}_{1}\right)\right)^{\alpha}\right)$ for $\alpha \geq 1$

(viii) $\mathscr{L}_{c_{1}}\left(\mathscr{M}_{1}\right)^{c}=\left(\mathscr{L}_{c_{j}}\left(\mathscr{M}_{1}\right)^{c}, \overline{\mathscr{L}_{c_{j}}}\left(\mathscr{M}_{1}\right)^{c}\right)$, where $\mathscr{L}_{c_{j}}\left(\mathscr{M}_{1}\right)^{c}$ are $\overline{\mathscr{L}}_{c_{j}}\left(\mathscr{M}_{1}\right)^{c}$, the complements of $\overline{\mathrm{q}-\mathrm{ROFS}} \mathrm{S}_{t} \mathrm{R}$ approximation operators $\mathscr{L}_{c_{j}}\left(\mathscr{M}_{1}\right)$ and $\overline{\mathscr{L}_{c_{j}}}\left(\mathscr{M}_{1}\right)$, that is, $\underline{\mathscr{L}_{c_{j}}}\left(\mathscr{M}_{1}\right)^{c}=\left(\underline{\vartheta_{i j}}, \overline{\beta_{i j}}\right)$ 
(ix) $\mathscr{L}\left(\mathscr{M}_{1}\right)=\mathscr{L}\left(\mathscr{M}_{2}\right)$ iff $\underline{\mathscr{L}}\left(\mathscr{M}_{1}\right)=\underline{\mathscr{L}}\left(\mathscr{M}_{2}\right)$ and $\overline{\mathscr{L}}$ $\left(\mathscr{M}_{1}\right)=\overline{\mathscr{L}}\left(\mathscr{M}_{2}\right)$

Definition 10. Let $\mathscr{L}_{c_{1}}\left(\mathscr{M}_{1}\right)=\left(\mathscr{L}_{c_{1}}\left(\mathscr{M}_{1}\right), \overline{\mathscr{L}_{c_{1}}}\left(\mathscr{M}_{1}\right)\right)=$ $\left(\left(\beta_{11}, \vartheta_{11}\right)\right),\left(\overline{\beta_{11}}, \overline{\vartheta_{11}}\right)$ be a q-ROFS $\bar{R}$ RN. Then, the score function is given as

$$
\begin{array}{r}
\operatorname{SC}\left(\mathscr{L}_{c_{1}}\left(\mathscr{M}_{1}\right)\right)=\frac{1}{2}\left(\underline{\beta_{11}^{q}}+\overline{\beta_{11}} q-\underline{\vartheta_{11}^{q}}-\overline{\vartheta_{11}} q\right), \\
\operatorname{SC}\left(\mathscr{L}_{c_{1}}\left(\mathscr{M}_{1}\right)\right) \in[-1,1] \text { and } q \geq 1 .
\end{array}
$$

The greater the score value, the greater the $\mathrm{q}-\mathrm{ROFS}_{t} \mathrm{RN}$.

Proposition 1. Let $(\mathscr{T}, \mathscr{E}, \mathscr{L})$ be q-ROFS approximation space. For any two $\mathscr{L}\left(\mathscr{M}_{1}\right)=\left(\mathscr{L}\left(\mathscr{M}_{1}\right), \overline{\mathscr{L}}\left(\mathscr{M}_{1}\right)\right)$ and $\mathscr{L}\left(\mathscr{M}_{2}\right)=\left(\underline{\mathscr{L}}\left(\mathscr{M}_{2}\right), \overline{\mathscr{L}}\left(\mathscr{M}_{2}\right)\right) q-R O F S_{t} R S$ s over a common universe set $Y$, then the following properties hold:

$$
\begin{aligned}
&\text { (i) }) \sim\left(\sim \mathscr{L}\left(\mathscr{M}_{1}\right)\right)=\mathscr{M}_{1} \text {, where } \sim \mathscr{L}\left(\mathscr{M}_{1}\right) \text { is the com- } \\
& \text { plement of } \mathscr{L}\left(\mathscr{M}_{1}\right), \quad \mathscr{L}\left(\mathscr{M}_{1}\right) \cup \mathscr{L}\left(\mathscr{M}_{2}\right) \\
&=\mathscr{L}\left(\mathscr{M}_{2}\right) \cup \mathscr{L}\left(\mathscr{M}_{1}\right), \mathscr{L}\left(\mathscr{M}_{1}\right) \cap \mathscr{L}\left(\mathscr{M}_{2}\right)=\mathscr{L}\left(\mathscr{M}_{2}\right) \\
& \cap \mathscr{L}\left(\mathscr{M}_{1}\right) \\
&\text { (ii) }) \sim\left(\mathscr{L}\left(\mathscr{M}_{1}\right) \cup \mathscr{L}\left(\mathscr{M}_{2}\right)\right)=\left(\sim \mathscr{L}\left(\mathscr{M}_{1}\right)\right) \cap(\sim \mathscr{L} \\
&\left.\left(\mathscr{M}_{2}\right)\right) \\
& \text { (iii) } \sim\left(\mathscr{L}\left(\mathscr{M}_{1}\right) \cap \mathscr{L}\left(\mathscr{M}_{2}\right)\right)=\left(\sim \mathscr{L}\left(\mathscr{M}_{1}\right)\right) \cup(\sim \mathscr{L} \\
& \\
&\left.\left(\mathscr{M}_{2}\right)\right) \\
& \text { (iv) } \text { If } \mathscr{M}_{1} \subseteq \mathscr{M}_{2}, \text { then } \mathscr{L}\left(\mathscr{M}_{1}\right) \subseteq \mathscr{L}\left(\mathscr{M}_{2}\right) \\
& \text { (v) } \mathscr{L}\left(\mathscr{M}_{1} \cup \mathscr{M}_{2}\right) \supseteq \mathscr{L}\left(\mathscr{M}_{1}\right) \cup \mathscr{L}\left(\mathscr{M}_{2}\right) \\
& \text { (vi) } \mathscr{L}\left(\mathscr{M}_{1} \cap \mathscr{M}_{2}\right) \subseteq \mathscr{L}\left(\mathscr{M}_{1}\right) \cap \mathscr{L}\left(\mathscr{M}_{2}\right)
\end{aligned}
$$

\section{4. q-Rung Orthopair Fuzzy Soft Rough Averaging (q-ROF $\left.S_{t} \mathbf{R A}\right)$ \\ Aggregation Operator}

This section is devoted to the analysis of $\mathrm{q}-\mathrm{ROFS}_{t} \mathrm{R}$ averaging aggregation operators such as q-ROFS $\mathrm{RWA}^{\mathrm{RW}}$ q-ROFS ROWA, and q-ROFS $_{t}$ RHA operators. We will present the fundamental properties of these operators in detail.

4.1. q-Rung Orthopair Fuzzy Soft Rough Weighted Averaging $\left(q-R O F S_{t} R W A\right)$ Operator

Definition 11. Let $\mathscr{L}_{c_{j}}\left(\mathscr{M}_{i}\right)=\left(\mathscr{L}_{c_{j}}\left(\mathscr{M}_{i}\right), \overline{\mathscr{L}_{c_{j}}}\left(\mathscr{M}_{i}\right)\right)(i=$ $1,2, \ldots, m, j=1,2, \ldots, n)$ be the collection of q-ROFS ${ }_{t}$ RNs. Let the weight vectors $t=\left(t_{1}, t_{2}, \ldots\right.$, $\left.t_{m}\right)^{T}$ and $v=\left(v_{1}, v_{2}, \ldots, v_{n}\right)^{T}$ for experts $s_{i}$ and parameters $c_{j}$ with $\sum_{i=1}^{m} t_{i}=1, \sum_{j=1}^{n} v_{j}=1$, and $0 \leq t_{i}, v_{j} \leq 1$, respectively. The q-ROFS ${ }_{t}$ RWA operator is defined as

$$
\begin{aligned}
\mathrm{q} & -\operatorname{ROFS}_{t} \operatorname{RWA}\left(\mathscr{L}_{c_{1}}\left(\mathscr{M}_{1}\right), \ldots, \mathscr{L}_{c_{n}}\left(\mathscr{M}_{m}\right)\right) \\
& =\left(\oplus_{j=1}^{n} v_{j}\left(\oplus_{i=1}^{m} t_{i} \underline{\mathscr{L}_{c_{j}}}\left(\mathscr{M}_{i}\right)\right), \oplus_{j=1}^{n} v_{j}\left(\oplus_{i=1}^{m} t_{i} \overline{\mathscr{L}_{c_{j}}}\left(\mathscr{M}_{i}\right)\right)\right) .
\end{aligned}
$$

In view of the above definition, Theorem 1 illustrates the aggregated result for $\mathrm{q}-\mathrm{ROFS}_{t} \mathrm{RWA}$.

Theorem 1. Let $\quad \mathscr{L}_{c_{j}}\left(\mathscr{M}_{i}\right)=\left(\mathscr{L}_{c_{j}}\left(\mathscr{M}_{i}\right), \overline{\mathscr{L}_{c_{j}}}\left(\mathscr{M}_{i}\right)\right)(i=$ $1,2, \ldots, m, j=1,2, \ldots, n)$ be the collection of $q-R O F S_{t} R N s$. Let the weight vectors $t=\left(t_{1}, t_{2}, \ldots, t_{m}\right)^{T}$ and $v=\left(v_{1}, v_{2}, \ldots, v_{n}\right)^{T}$ for experts $s_{i}$ and parameters $c_{j}$ with $\sum_{i=1}^{m} t_{i}=1, \sum_{j=1}^{n} v_{j}=1$ and $0 \leq t_{i}, v_{j} \leq 1$, respectively. Then, $q-R^{2} \mathrm{FS}_{t} \mathrm{RWA}$ operator is given as

$$
\begin{aligned}
& \mathrm{q}-\operatorname{ROFS}_{t} \operatorname{RWA}\left(\mathscr{L}_{c_{1}}\left(\mathscr{M}_{1}\right), \ldots, \mathscr{L}_{c_{n}}\left(\mathscr{M}_{m}\right)\right)=\left[\oplus_{j=1}^{n} v_{j}\left(\oplus_{i=1}^{m} t_{i} \mathscr{L}_{c_{j}}\left(\mathscr{M}_{i}\right)\right), \oplus_{j=1}^{n} v_{j}\left(\oplus_{i=1}^{m} t_{i} \overline{\mathscr{L}_{c_{j}}}\left(\mathscr{M}_{i}\right)\right)\right]
\end{aligned}
$$

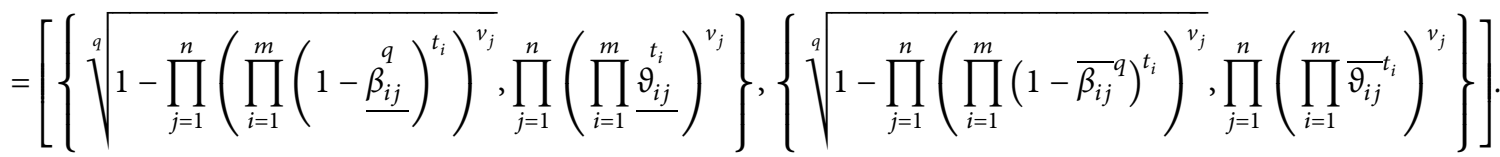

Proof. To get the required proof, we will use the method of mathematical induction.
As by operational law,

$$
\begin{aligned}
& \mathscr{L}_{c_{1}}\left(\mathscr{M}_{1}\right) \oplus \mathscr{L}_{c_{1}}\left(\mathscr{M}_{2}\right)=\left(\left(\underline{\beta_{11}}, \underline{\vartheta_{11}}\right) \oplus\left(\underline{\beta_{12}}, \underline{\vartheta_{12}}\right),\left(\overline{\beta_{11}}, \overline{\vartheta_{11}}\right) \oplus\left(\overline{\beta_{12}}, \overline{\vartheta_{12}}\right)\right)
\end{aligned}
$$

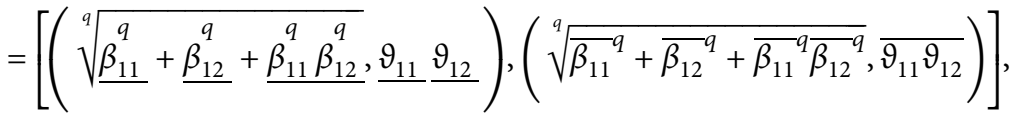

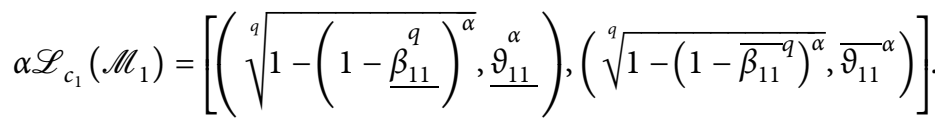


Suppose the result is true for $m=2$ and $n=2$; that is, $\quad$ Now, consider

$$
\begin{aligned}
& \mathrm{q}-\operatorname{ROFS}_{t} \operatorname{RWA}\left(\mathscr{L}_{c_{j}}\left(\mathscr{M}_{i}\right), \mathscr{L}_{c_{j}}\left(\mathscr{M}_{i}\right)\right) \\
& =\left(\oplus_{j=1}^{2} v_{j}\left(\oplus_{i=1}^{2} t_{i} \underline{\mathscr{L}_{c_{j}}}\left(\mathscr{M}_{i}\right)\right), \oplus_{j=1}^{2} v_{j}\left(\oplus_{i=1}^{2} t_{i} \overline{\mathscr{L}_{c_{j}}}\left(\mathscr{M}_{i}\right)\right)\right) .
\end{aligned}
$$

$$
\begin{aligned}
& \mathrm{q}-\operatorname{ROFS}_{t} \operatorname{RWA}\left(\mathscr{L}_{c_{j}}\left(\mathscr{M}_{i}\right), \mathscr{L}_{c_{j}}\left(\mathscr{M}_{i}\right)\right)=\left[\oplus_{j=1}^{2} v_{j}\left(\oplus_{i=1}^{2} t_{i} \underline{\mathscr{L}_{c_{j}}}\left(\mathscr{M}_{i}\right)\right), \oplus_{j=1}^{2} v_{j}\left(\oplus_{i=1}^{2} t_{i} \overline{\mathscr{L}}_{c_{j}}\left(\mathscr{M}_{i}\right)\right)\right] \\
& =\left[\left\{v_{1}\left(t_{1} \underline{\mathscr{L}_{c_{1}}}\left(\mathscr{M}_{1}\right) \oplus t_{2} \underline{\mathscr{L}_{c_{1}}}\left(\mathscr{M}_{2}\right)\right) \oplus v_{2}\left(t_{1} \underline{\mathscr{L}_{c_{2}}}\left(\mathscr{M}_{1}\right) \oplus t_{2} \underline{\mathscr{L}_{c_{2}}}\left(\mathscr{M}_{2}\right)\right)\right\},\right. \\
& \left.\left\{v_{1}\left(t_{1} \overline{\mathscr{L}_{c_{j}}}\left(\mathscr{M}_{1}\right) \oplus t_{2} \overline{\mathscr{L}_{c_{j}}}\left(\mathscr{M}_{2}\right)\right) \oplus v_{2}\left(t_{1} \overline{\mathscr{L}_{c_{j}}}\left(\mathscr{M}_{1}\right) \oplus t_{2} \overline{\mathscr{L}_{c_{j}}}\left(\mathscr{M}_{2}\right)\right)\right\}\right] \text {, }
\end{aligned}
$$

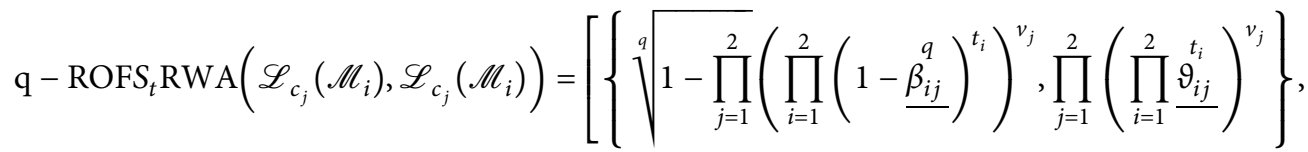

$$
\begin{aligned}
& \left\{\left(\sqrt[q]{1-\prod_{j=1}^{2}}\left(\prod_{i=1}^{2}\left(1-\overline{\beta_{i j}}{ }^{q}\right)^{t_{i}}\right)^{v_{j}}, \prod_{j=1}^{2}\left(\prod_{i=1}^{2} \overline{\bar{\vartheta}_{i j}}{ }^{t_{i}}\right)^{v_{j}}\right)\right\} .
\end{aligned}
$$

The result is true for $m=2$ and $n=2$.

Now, consider the result is for $n=k_{1}$ and $m=k_{2}$ :

$$
\begin{aligned}
& \mathrm{q}-\operatorname{ROFS}_{t} \operatorname{RWA}\left(\mathscr{L}_{c_{j}}\left(\mathscr{M}_{i}\right), \mathscr{L}_{c_{j}}\left(\mathscr{M}_{i}\right), \ldots, \mathscr{L}_{c_{k_{1}}}\left(\mathscr{M}_{k_{2}}\right)\right)
\end{aligned}
$$

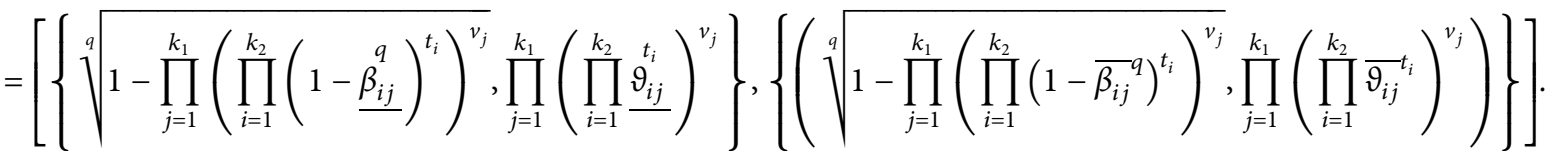

Suppose the result holds for $n=k_{1}+1$ and $m=k_{2}+1$, so we have

$$
\begin{aligned}
& \mathrm{q}-\operatorname{ROFS}_{t} \operatorname{RWA}\left[\left(\mathscr{L}_{c_{j}}\left(\mathscr{M}_{i}\right), \mathscr{L}_{c_{j}}\left(\mathscr{M}_{i}\right), \ldots, \mathscr{L}_{c_{k_{1}}}\left(\mathscr{M}_{k_{2}}\right)\right), \mathscr{L}_{c_{k_{1}+1}}\left(\mathscr{M}_{k_{2}+1}\right)\right] \\
& =\left[\oplus_{j=1}^{k_{1}} v_{j}\left(\oplus_{i=1}^{k_{2}} t_{i} \mathscr{L}_{c_{j}}\left(\mathscr{M}_{i}\right)\right) \oplus v_{k_{1}+1}\left(t_{k_{2}+1} \underline{\mathscr{L}_{c_{k_{1}+1}}}\left(\mathscr{M}_{k_{2}+1}\right)\right), \oplus_{j=1}^{k_{1}} v_{j}\left(\oplus_{i=1}^{k_{2}} t_{i} \overline{\mathscr{L}_{c_{j}}}\left(\mathscr{M}_{i}\right)\right) \oplus v_{k_{1}+1}\left(t_{k_{2}+1} \overline{\mathscr{L}}_{c_{k_{1}+1}}\left(\mathscr{M}_{k_{2}+1}\right)\right)\right]
\end{aligned}
$$

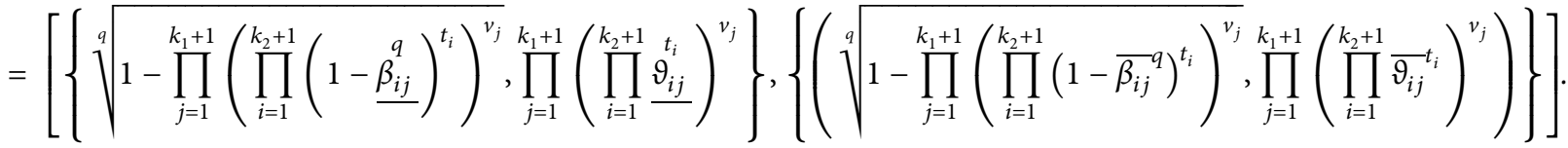

This implies the result is true for $n=k_{1}+1$ and $m=k_{2}+1$. Therefore, the result holds for all $m, n \geq 1$.

Since it is clear that $\mathscr{L}_{c_{j}}\left(\mathscr{M}_{i}\right)$ and $\overline{\mathscr{L}_{c_{j}}}\left(\mathscr{M}_{i}\right)$ are qROFNs, by Definition 7, we have that $\oplus_{j=1}^{n} v_{j}\left(\oplus_{i=1}^{m} t_{i} \mathscr{L}_{c_{j}}\left(\mathscr{M}_{i}\right)\right)$ and $\oplus_{j=1}^{n} v_{j}\left(\oplus_{i=1}^{m} t_{i} \overline{\mathscr{L}_{c_{j}}}\left(\mathscr{M}_{i}\right)\right)$ are

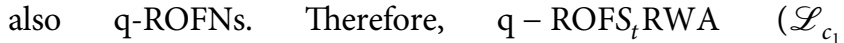
$\left.\left(\mathscr{M}_{1}\right), \ldots, \mathscr{L}_{c_{n}}\left(\mathscr{M}_{m}\right)\right)$ is also a q-ROFS $\mathrm{R}_{t} \mathrm{RN}$ in approximation space $(\mathscr{T}, \mathscr{E}, \mathscr{L})$.

Example 2. Let $Y=\left\{s_{1}, s_{2}, s_{3}\right\}$ be a set and $\mathscr{M}=\left\{c_{1}, c_{2}\right\} \subseteq \mathscr{E}$ a set of parameters with weight vector $t=(0.25,0.3,0.45)^{T}$ for 
$s_{i}(i=1,2,3)$ and $v=(0.55,0.45)$ for $c_{j}(j=1,2)$. Then,

$\mathrm{q}-\mathrm{ROFS}_{t} \mathrm{RNs}$ is given in Table 3 :

$$
\begin{aligned}
\mathrm{q} & -\operatorname{ROFS}_{t} \operatorname{RWA}\left(\mathscr{L}_{c_{1}}\left(\mathscr{M}_{1}\right), \ldots, \mathscr{L}_{c_{n}}\left(\mathscr{M}_{m}\right)\right)=\left[\oplus_{j=1}^{2} v_{j}\left(\oplus_{i=1}^{3} t_{i} \mathscr{L}_{c_{j}}\left(\mathscr{M}_{i}\right)\right), \oplus_{j=1}^{2} v_{j}\left(\oplus_{i=1}^{3} t_{i} \overline{\mathscr{L}}_{c_{j}}\left(\mathscr{M}_{i}\right)\right)\right] \\
= & {\left[\left\{\sqrt[3]{1-\left(\left[\left(1-0.9^{3}\right)^{0.25}\left(1-0.7^{3}\right)^{0.25}\left(1-0.29^{3}\right)^{0.45}\right]^{0.55}\left[\left(1-0.55^{3}\right)^{0.25}\left(1-0.92^{3}\right)^{0.25}\left(1-0.4^{3}\right)^{0.45}\right]^{0.45}\right),}\right.\right.} \\
& \left.\left(0.3^{0.25} 0.1^{0.3} 0.25^{0.45}\right)^{0.55}\left(0.2^{0.25} 0.3^{0.3} 0.85^{0.45}\right)^{0.45}\right\}, \\
& \left\{\sqrt[3]{1-\left(\left[\left(1-0.4^{3}\right)^{0.25}\left(1-0.2^{3}\right)^{0.25}\left(1-0.65^{3}\right)^{0.45}\right]^{0.55}\left[\left(1-0.76^{3}\right)^{0.25}\left(1-0.6^{3}\right)^{0.25}\left(1-0.88^{3}\right)^{0.45}\right]^{0.45}\right),}\right. \\
& \left.\left.\left(0.4^{0.25} 0.75^{0.3} 0.15^{0.45}\right)^{0.55}\left(0.14^{0.25} 0.3^{0.3} 0.12^{0.45}\right)^{0.45}\right\}\right] \\
= & {[(0.831432,0.255487),(0.72581,0.26258)] . }
\end{aligned}
$$

From the analysis of Theorem 1, some related characteristics of $\mathrm{q}-\mathrm{ROFS}_{t} \mathrm{RWA}$ operator are given as follows:

Theorem 2. Let $\quad \mathscr{L}_{c_{j}}\left(\mathscr{M}_{i}\right)=\left(\mathscr{L}_{c_{j}}\left(\mathscr{M}_{i}\right), \overline{\mathscr{L}_{c_{j}}}\left(\mathscr{M}_{i}\right)\right)(i=$ $1,2, \ldots, m, j=1,2, \ldots, n)$ be the collection of $q-R O F S_{t} R N s$. Let the weight vectors $t=\left(t_{1}, t_{2}, \ldots, t_{m}\right)^{T}$ and $v=\left(v_{1}, v_{2}\right.$, $\left.\ldots, v_{n}\right)^{T}$ for experts $s_{i}$ and parameters $c_{j}$ with $\sum_{i=1}^{m} t_{i}=1$, $\sum_{j=1}^{n} v_{j}=1$ and $0 \leq t_{i}, v_{j} \leq 1$, respectively. Then, the following properties hold for q-ROFS $S_{t}$ RWA operator:

(i) (Idempotency): if $\mathscr{L}_{c_{i}}\left(\mathscr{M}_{i}\right)=\mathscr{P}_{c}(\mathcal{N}) \quad$ (for all $i=$ $1,2, \ldots, \quad$ mand $j=1,2, \ldots n)$, where $\mathscr{P}_{c}(\mathscr{M})=$ $\left(\underline{\mathscr{P}_{c}}(\mathscr{M}), \overline{\mathscr{P}}_{c}(\mathscr{M})\right)=((\underline{b}, \underline{d}),(\bar{b}, \bar{d}))$, then

$\mathrm{q}-\operatorname{ROFS}_{\mathrm{t}} \operatorname{RWA}\left(\mathscr{L}_{c_{1}}\left(\mathscr{M}_{1}\right), \mathscr{L}_{c_{2}}\left(\mathscr{M}_{2}\right), \ldots, \mathscr{L}_{c_{n}}\left(\mathscr{M}_{m}\right)\right)$

$=\mathscr{P}_{c}(\mathscr{M})$.

(ii) (Boundedness): let $\left(\mathscr{L}_{c_{j}}\left(\mathscr{M}_{i}\right)\right)^{-}=\left(\min _{j} \min _{i} \mathscr{L}_{c_{j}}\right.$ $\left.\left(\mathscr{M}_{i}\right), \max _{j} \max _{i} \overline{\mathscr{L}_{c_{j}}}\left(\mathscr{M}_{i}\right)\right)$ and $\left(\mathscr{L}_{c_{j}}\left(\mathscr{M}_{i}\right)\right)^{+} \stackrel{{ }^{c_{j}}}{=}$ $\left(\max _{j} \max _{i} \mathscr{L}_{c_{j}}\left(\mathscr{M}_{i}\right), \min _{j} \min _{i} \overline{\mathscr{L}}_{c_{j}}\left(\mathscr{M}_{i}\right)\right)$. Then,

$$
\begin{gathered}
\left(\mathscr{L}_{c_{j}}\left(\mathscr{M}_{i}\right)\right)^{-} \leq \mathrm{q}-\operatorname{ROFS}_{t} \operatorname{RWA}\left(\mathscr{L}_{c_{1}}\left(\mathscr{M}_{1}\right), \mathscr{L}_{c_{2}}\right. \\
\left.\left(\mathscr{M}_{2}\right), \ldots, \mathscr{L}_{c_{n}}\left(\mathscr{M}_{m}\right)\right) \leq\left(\mathscr{L}_{c_{j}}\left(\mathscr{M}_{i}\right)\right)^{+} .
\end{gathered}
$$

(iii) (Monotonicity): let $\mathscr{P}_{c_{j}}\left(\mathcal{N}_{i}\right)=\left(\mathscr{P}_{c_{j}}\left(\mathcal{N}_{i}\right), \overline{\mathscr{P}_{c_{j}}}\right.$ $\left.\left(\mathcal{N}_{i}\right)\right)(i=1,2, \ldots, m, j=1,2, \ldots, \bar{n})$ be another collection of $q-R O F S_{t} R N s$ such that $\mathscr{P}_{c_{j}}$ $\left(\mathcal{N}_{i}\right) \leq \mathscr{L}_{c_{j}}\left(\mathscr{M}_{i}\right)$ and $\overline{\mathscr{P}_{c_{j}}}\left(\mathscr{N}_{i}\right) \leq \overline{\mathscr{L}_{c_{j}}}\left(\mathscr{M}_{i}\right)$. Then,

$$
\begin{aligned}
\mathrm{q} & -\operatorname{ROFS}_{t} \operatorname{RWA}\left(\mathscr{P}_{c_{1}}\left(\mathscr{N}_{1}\right), \mathscr{P}_{c_{2}}\left(\mathscr{N}_{2}\right), \ldots, \mathscr{P}_{c_{n}}\left(\mathscr{N}_{m}\right)\right) \\
& \leq \mathrm{q}-\operatorname{ROFS}{ }_{t} \operatorname{RWA}\left(\mathscr{L}_{c_{1}}\left(\mathscr{M}_{1}\right), \mathscr{L}_{c_{2}}\left(\mathscr{M}_{2}\right), \ldots, \mathscr{L}_{c_{n}}\left(\mathscr{M}_{m}\right)\right) .
\end{aligned}
$$

(iv) (Shift_ invariance): let $\quad \mathscr{P}_{c}(\mathcal{N})=\left(\mathscr{P}_{c}\right.$ $\left.(\mathscr{N}), \overline{\mathscr{P}}_{c}(\mathcal{N})\right)=((\underline{b}, \underline{d}),(\bar{b}, \bar{d}))$ be any other $q-R^{2} \mathrm{FS}_{t} R N$. Then,

$$
\begin{aligned}
\mathrm{q}- & \operatorname{ROFS} S_{t} \operatorname{RWA}\left(\mathscr{L}_{c_{1}}\left(\mathscr{M}_{1}\right) \oplus \mathscr{P}_{c}(\mathcal{N}), \mathscr{L}_{c_{2}}\left(\mathscr{M}_{2}\right)\right. \\
& \left.\oplus \mathscr{P}_{c}(\mathcal{N}), \ldots, \mathscr{L}_{c_{n}}\left(\mathscr{M}_{m}\right) \oplus \mathscr{P}_{c}(\mathcal{N})\right) \\
=\mathrm{q}- & \operatorname{ROFS} S_{t} \operatorname{RWA}\left(\mathscr{L}_{c_{1}}\left(\mathscr{M}_{1}\right), \mathscr{L}_{c_{2}}\left(\mathscr{M}_{2}\right), \ldots, \mathscr{L}_{c_{n}}\left(\mathscr{M}_{m}\right)\right) \\
& \oplus \mathscr{P}_{c}(\mathscr{N}) .
\end{aligned}
$$

(v) (Homogeneity): for any real number $\lambda>0$,

$$
\begin{aligned}
\mathrm{q} & -\operatorname{ROFS}_{t} \operatorname{RWA}\left(\lambda \mathscr{L}_{c_{1}}\left(\mathscr{M}_{1}\right), \lambda \mathscr{L}_{\mathcal{c}_{2}}\left(\mathscr{M}_{2}\right), \ldots, \lambda \mathscr{L}_{c_{n}}\left(\mathscr{M}_{m}\right)\right) \\
& =\lambda \mathrm{q}-\operatorname{ROFS}_{t} \operatorname{RWA}\left(\mathscr{L}_{c_{1}}\left(\mathscr{M}_{1}\right), \mathscr{L}_{c_{2}}\left(\mathscr{M}_{2}\right), \ldots, \mathscr{L}_{c_{n}}\left(\mathscr{M}_{m}\right)\right) .
\end{aligned}
$$

Remark 2

(a) If $q=1$, so, in this case, the developed $\mathrm{q}-\mathrm{ROFS}_{t}$ RWA operator reduces to IFS ${ }_{t}$ RWA operator

(b) If $q=2$, so, in this case, the developed $\mathrm{q}-\mathrm{ROFS}_{t}$ RWA operator reduces to $\mathrm{PFS}_{t} \mathrm{RWA}$ operator 
TABLE 3: Tabular representation of $\mathscr{L}_{c_{j}}\left(\mathscr{M}_{i}\right)=\left(\mathscr{L}_{c_{j}}\left(\mathscr{M}_{i}\right)\right.$, $\left.\overline{\mathscr{L}_{c_{j}}}\left(\mathscr{M}_{i}\right)\right)$.

\begin{tabular}{lcc}
\hline $\mathscr{L}$ & $c_{1}$ & $c_{2}$ \\
\hline$s_{1}$ & $((0.9,0.3)),(0.8,0.4)$ & $((0.55,0.2)),(0.76,0.14)$ \\
$s_{2}$ & $((0.7,0.1),(0.2,0.75))$ & $((0.92,0.3),(0.6,0.3))$ \\
$s_{3}$ & $((0.92,0.25),(0.65,0.15))$ & $((0.4,0.85),(0.88,0.12))$ \\
\hline
\end{tabular}

(c) If the soft parameter $c_{1}$ is only one (means $n=1$ ), then, in this case, the developed q-ROFS RWA $^{-}$ operator reduces to q-ROFRWA operator

4.2. q-Rung Orthopair Fuzzy Soft Rough Ordered Weighted Averaging ( $q$-ROFS ROWA $_{t}$ Operator. This subsection presents the detailed study of q-ROFS ${ }_{t}$ ROWA operator and some of its desirable characteristics such as Idempotency, Boundedness, and Monotonicity. The basic advantage of

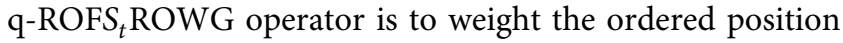
of the q-ROFVs instead of weighting the values themselves.

Definition 12. Let $\mathscr{L}_{c_{j}}\left(\mathscr{M}_{i}\right)=\left(\mathscr{L}_{c_{j}}\left(\mathscr{M}_{i}\right), \overline{\mathscr{L}_{c_{j}}}\left(\mathscr{M}_{i}\right)\right)(i=$ $1,2, \ldots, m, j=1,2, \ldots, n)$ be the collection of q-ROFS ${ }_{t}$ RNs. Let the weight vectors $t=\left(t_{1}, t_{2}, \ldots, t_{m}\right)^{T}$ and $v=$ $\left(v_{1}, v_{2}, \ldots, v_{n}\right)^{T}$ for experts $s_{i}$ and parameters $c_{j}$ with
TABLE 4: Tabular representation of $\mathscr{L}_{\delta c_{j}}\left(\mathscr{M}_{i}\right)=\left(\mathscr{L}_{\delta c_{j}}\left(\mathscr{M}_{i}\right)\right.$, $\left.\overline{\mathscr{L}_{\delta c_{j}}}\left(\mathscr{M}_{i}\right)\right)$

\begin{tabular}{ccc}
\hline $\mathscr{L}$ & $c_{1}$ & $c_{2}$ \\
\hline$s_{1}$ & $((0.9,0.2),(0.8,0.4))$ & $((0.92,0.3),(0.6,0.3))$ \\
$s_{2}$ & $((0.92,0.25),(0.65,0.15))$ & $((0.55,0.2),(0.76,0.14))$ \\
$s_{3}$ & $((0.7,0.1),(0.2,0.75))$ & $((0.4,0.85),(0.88,0.12))$ \\
\hline
\end{tabular}

$\sum_{i=1}^{m} t_{i}=1, \sum_{j=1}^{n} v_{j}=1$, and $0 \leq t_{i}, v_{j} \leq 1$, respectively. The q-ROFS $S_{t}$ ROWA operator is defined as

$$
\begin{aligned}
\mathrm{q} & -\operatorname{ROFS}_{t} \operatorname{ROWA}\left(\mathscr{L}_{c_{1}}\left(\mathscr{M}_{1}\right), \ldots, \mathscr{L}_{c_{n}}\left(\mathscr{M}_{m}\right)\right) \\
& =\left(\oplus_{j=1}^{n} v_{j}\left(\oplus_{i=1}^{m} t_{i} \underline{\mathscr{L}_{\delta c_{j}}}\left(\mathscr{M}_{i}\right)\right), \oplus_{j=1}^{n} v_{j}\left(\oplus_{i=1}^{m} t_{i} \overline{\mathscr{L}_{\delta c_{j}}}\left(\mathscr{M}_{i}\right)\right)\right) .
\end{aligned}
$$

In view of the above definition, the aggregated result for

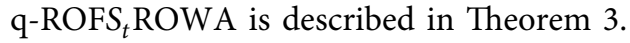

Theorem 3. Let $\quad \mathscr{L}_{c_{j}}\left(\mathscr{M}_{i}\right)=\left(\mathscr{L}_{c_{j}}\left(\mathscr{M}_{i}\right), \overline{\mathscr{L}_{c_{j}}}\left(\mathscr{M}_{i}\right)\right)(i=$ $1,2, \ldots, m, j=1,2, \ldots, n)$ be the collection of $q-R O F S_{t} R N s$. Let the weight vectors $t=\left(t_{1}, t_{2}, \ldots, t_{m}\right)^{T}$ and $v$ $=\left(v_{1}, v_{2}, \ldots, v_{n}\right)^{T}$ for experts $s_{i}$ and parameters $c_{j}$ with $\sum_{i=1}^{m} t_{i}=1, \sum_{j=1}^{n} v_{j}=1$, and $0 \leq t_{i}, v_{j} \leq 1$, respectively. Then, $q-$ ROFS $_{t}$ ROWA operator is given as

$$
\begin{aligned}
& \mathrm{q}-\operatorname{ROFS}_{t} \operatorname{ROWA}\left(\mathscr{L}_{c_{1}}\left(\mathscr{M}_{1}\right), \ldots, \mathscr{L}_{c_{n}}\left(\mathscr{M}_{m}\right)\right)=\left(\oplus_{j=1}^{n} v_{j}\left(\oplus_{i=1}^{m} t_{i} \underline{\mathscr{L}_{\delta c_{j}}}\left(\mathscr{M}_{i}\right)\right), \oplus_{j=1}^{n} v_{j}\left(\oplus_{i=1}^{m} t_{i} \overline{\mathscr{L}}_{\delta c_{j}}\left(\mathscr{M}_{i}\right)\right)\right)
\end{aligned}
$$

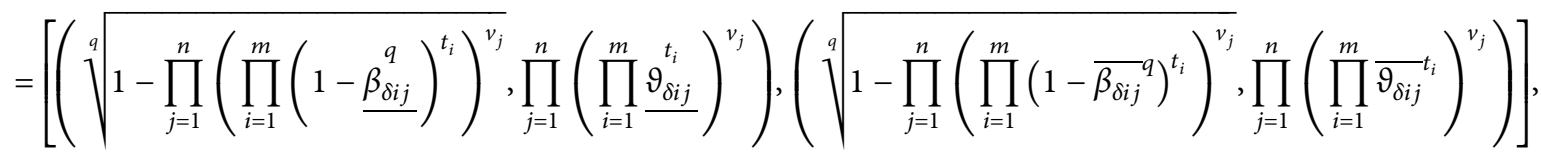

where $\mathscr{L}_{\delta c_{j}}\left(\mathscr{M}_{i}\right)=\left(\mathscr{L}_{\delta c_{j}}\left(\mathscr{M}_{i}\right), \overline{\mathscr{L}_{\delta c_{j}}}\left(\mathscr{M}_{i}\right)\right)$ denotes the largest value of the permutation from ith row and jth column of $\mathscr{U}_{i}$ the collection $i \times j$ q-ROFS $R N s$ $\mathscr{L}_{c_{j}}\left(\mathscr{M}_{i}\right)=\left(\mathscr{L}_{c_{j}}\left(\mathscr{M}_{i}\right), \mathscr{L}_{c_{j}}\left(\mathscr{M}_{i}\right)\right)$.

Example 3. Consider Table 3 of Example 2, for the collection

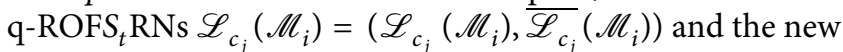
ordered of tabular representation of $\mathscr{L}_{c_{j}}\left(\mathscr{M}_{i}\right)$ through score function is given in Table 4 .

$\quad$ Now, $\quad \mathrm{q}-\mathrm{ROFS}_{t} \operatorname{ROWA}\left(\mathscr{L}_{c_{1}}\left(\mathscr{M}_{1}\right), \ldots, \mathscr{L}_{c_{n}}\right.$ $\left.\left(\mathscr{M}_{m}\right)\right)=\left[\oplus_{j=1}^{2} v_{j}\left(\oplus_{i=1}^{3} t_{i} \mathscr{L}_{\delta c_{j}}\left(\mathscr{M}_{i}\right)\right), \oplus_{j=1}^{2} v_{j}\left(\oplus_{i=1}^{3} t_{i} \overline{\mathscr{L}_{\delta c_{j}}}\right.\right.$ $\left.\left.\left(\mathscr{M}_{i}\right)\right)\right]$

$$
\begin{aligned}
\mathrm{q}- & \operatorname{ROFS}_{t} \operatorname{ROWA}\left(\mathscr{L}_{c_{1}}\left(\mathscr{M}_{1}\right), \ldots, \mathscr{L}_{c_{n}}\left(\mathscr{M}_{m}\right)\right) \\
& =((0.838595,0.261642),(0.727318,0.255189)) .
\end{aligned}
$$

From the analysis of Theorem 3, the following desirable

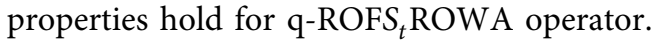

Theorem 4. Let $\quad \mathscr{L}_{c_{j}}\left(\mathscr{M}_{i}\right)=\left(\mathscr{L}_{c_{j}}\left(\mathscr{M}_{i}\right), \overline{\mathscr{L}_{c_{j}}}\left(\mathscr{M}_{i}\right)\right)(i=$ $1,2, \ldots, m, j=1,2, \ldots, n)$ be the collection of $q-R^{2} F_{t} R N s$. Let the weight vectors $t=\left(t_{1}, t_{2}, \ldots, t_{m}\right)^{T}$ and $v=$ $\left(v_{1}, v_{2}, \ldots, v_{n}\right)^{T}$ for experts $s_{i}$ and parameters $c_{j}$ with $\sum_{i=1}^{m} t_{i}=1, \sum_{j=1}^{n} v_{j}=1$, and $0 \leq t_{i}, v_{j} \leq 1$, respectively. Then, the following properties hold for $q-R_{O F S} R O W A$ operator:

(i) (Idempotency): if $\mathscr{L}_{\delta c_{j}}\left(\mathscr{M}_{i}\right)=\mathscr{P}_{c}(\mathcal{N}) \quad$ (for all $i=$ $1,2, \ldots$, m and $j=1,2, \ldots, n)$, where $\mathscr{P}_{c}(\mathscr{M})=\left(\mathscr{P}_{c}\right.$ $\left.(\mathscr{M}), \overline{\mathscr{P}}_{c}(\mathscr{M})\right)=((\underline{b}, \underline{d}),(\bar{b}, \bar{d}))$, then

$\mathrm{q}-\operatorname{ROFS}_{t} \operatorname{ROWA}\left(\mathscr{L}_{c_{1}}\left(\mathscr{M}_{1}\right), \mathscr{L}_{c_{2}}\left(\mathscr{M}_{2}\right), \ldots, \mathscr{L}_{c_{n}}\left(\mathscr{M}_{m}\right)\right)=\mathscr{P}_{c}(\mathscr{M})$.

(ii) (Boundedness): let $\left(\mathscr{L}_{\delta c}\left(\mathscr{M}_{i}\right)\right)^{-}=\left(\min _{j} \min _{i}\right.$ $\left.\mathscr{L}_{\delta c_{j}}\left(\mathscr{M}_{i}\right), \max _{j} \max _{i} \overline{\mathscr{L}_{\delta c_{j}}}\left(\mathscr{M}_{i}\right)\right)$ and $\left(\mathscr{L}_{\delta c_{j}}\left(\mathscr{M}_{i}\right)\right)^{+}$

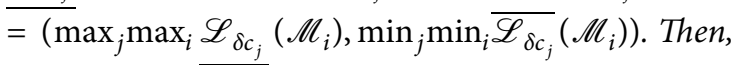

$$
\begin{gathered}
\left(\mathscr{L}_{\delta c_{j}}\left(\mathscr{M}_{i}\right)\right)^{-} \leq \mathrm{q}-\operatorname{ROFS}_{t} \operatorname{ROWA}\left(\mathscr{L}_{c_{1}}\left(\mathscr{M}_{1}\right),\right. \\
\left.\mathscr{L}_{c_{2}}\left(\mathscr{M}_{2}\right), \ldots, \mathscr{L}_{c_{n}}\left(\mathscr{M}_{m}\right)\right) \leq\left(\mathscr{L}_{\delta c_{j}}\left(\mathscr{M}_{i}\right)\right)^{+} .
\end{gathered}
$$

(iii) (Monotonicity): let $\quad \mathscr{P}_{c_{j}}\left(\mathscr{N}_{i}\right)=\left(\mathscr{P}_{c_{j}}\left(\mathscr{N}_{i}\right), \overline{\mathscr{P}_{c_{j}}}\right.$ $\left.\left(\mathcal{N}_{i}\right)\right)(i=1,2, \ldots, m, j=1,2, \ldots, n) \overline{\text { be }}$ another 


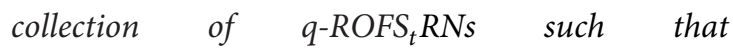

$$
\begin{aligned}
& \underline{\mathscr{P}_{c_{j}}}\left(\mathcal{N}_{i}\right) \leq \underline{\mathscr{L}_{c_{j}}}\left(\mathscr{M}_{i}\right) \text { and } \overline{\mathscr{P}_{c_{j}}}\left(\mathscr{N}_{i}\right) \leq \overline{\mathscr{L}_{c_{j}}}\left(\mathscr{M}_{i}\right) \text {. } \\
& \text { Then, } \\
& \mathrm{q}-\operatorname{ROFS} S_{t} \operatorname{ROWA}\left(\mathscr{P}_{c_{1}}\left(\mathscr{M}_{1}\right), \mathscr{P}_{c_{2}}\left(\mathscr{M}_{2}\right), \ldots, \mathscr{P}_{c_{n}}\left(\mathscr{M}_{m}\right)\right) \\
& \leq \mathrm{q}-\operatorname{ROFS}{ }_{t} \operatorname{ROWA}\left(\mathscr{L}_{c_{1}}\left(\mathscr{M}_{1}\right), \mathscr{L}_{c_{2}}\left(\mathscr{M}_{2}\right), \ldots, \mathscr{L}_{c_{n}}\left(\mathscr{M}_{m}\right)\right) \text {. }
\end{aligned}
$$

(iv) (Shift invariance): let $\mathscr{P}_{c}(\mathscr{N})=\left(\mathscr{P}_{c}(\mathcal{N})\right.$, $\left.\overline{\mathscr{P}}_{c}(\mathcal{N})\right)=((\underline{b}, \underline{d}),(\bar{b}, \bar{d}))$ be any other $q-$ ROFS $_{t} R N$. Then,

$$
\begin{aligned}
\mathrm{q}- & \operatorname{ROFS}_{t} \operatorname{ROWA}\left(\mathscr{L}_{c_{1}}\left(\mathscr{M}_{1}\right) \oplus \mathscr{P}_{c}(\mathscr{N}), \mathscr{L}_{c_{2}}\left(\mathscr{M}_{2}\right)\right. \\
& \left.\oplus \mathscr{P}_{c}(\mathcal{N}), \ldots, \mathscr{L}_{c_{n}}\left(\mathscr{M}_{m}\right) \oplus \mathscr{P}_{c}(\mathcal{N})\right) \\
= & \mathrm{q}-\operatorname{ROFS}_{t} \operatorname{ROWA}\left(\mathscr{L}_{c_{1}}\left(\mathscr{M}_{1}\right), \mathscr{L}_{c_{2}}\left(\mathscr{M}_{2}\right), \ldots, \mathscr{L}_{c_{n}}\left(\mathscr{M}_{m}\right)\right) \\
& \oplus \mathscr{P}_{c}(\mathcal{N}) .
\end{aligned}
$$

(v) (Homogeneity): for any real number $\lambda>0$,

$$
\begin{gathered}
\mathrm{q}-\operatorname{ROFS}_{t} \operatorname{ROWA}\left(\lambda \mathscr{L}_{c_{1}}\left(\mathscr{M}_{1}\right), \lambda \mathscr{L}_{c_{2}}\left(\mathscr{M}_{2}\right), \ldots,\right. \\
\left.\lambda \mathscr{L}_{c_{n}}\left(\mathscr{M}_{m}\right)\right)=\lambda \mathrm{q}-\mathrm{ROFS}_{t} \operatorname{ROWA} \\
\left(\mathscr{L}_{c_{1}}\left(\mathscr{M}_{1}\right), \mathscr{L}_{c_{2}}\left(\mathscr{M}_{2}\right), \ldots, \mathscr{L}_{c_{n}}\left(\mathscr{M}_{m}\right)\right) .
\end{gathered}
$$

Remark 3

(a) If $q=1$, then the developed $\mathrm{q}-\mathrm{ROFS}_{t} \mathrm{ROWA}$ operator reduces to $\mathrm{IFS}_{t} \mathrm{ROWA}$ operator (b) If $q=2$, then the developed q-ROFS,ROWA operator reduces to $\mathrm{PFS}_{t} \mathrm{ROWA}$ operator

(c) If the soft parameter $c_{1}$ is one (means $n=1$ ), then the developed q-ROFS $\mathrm{ROWA}_{t}$ operator reduces to q-ROFROWA operator

4.3. q-Rung Orthopair Fuzzy Soft Rough Hybrid Averaging ( $q$ $\left.R O F S_{t} R H A\right)$ Operator. From the analysis of Definitions 11 and 12 , it is clear that the $\mathrm{q}-\mathrm{ROFS}_{t} \mathrm{RWA}$ operator weights only the q-ROFVs, while q-ROFS ${ }_{t}$ ROWA operator weights the ordered position of the q-ROFVs instead of weighting the values themselves. To overcome this limitation and motivated by the idea of combining weighted averaging and the ordered weighted averaging by using the combined

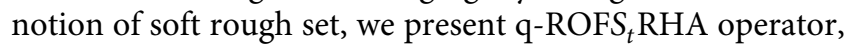
which weights both the given q-ROFV and its ordered position. The basic desirable properties of the developed operator are presented in detail.

Definition 13. Let $\mathscr{L}_{c_{j}}\left(\mathscr{M}_{i}\right)=\left(\mathscr{L}_{c_{j}}\left(\mathscr{M}_{i}\right), \overline{\mathscr{L}_{c_{j}}}\left(\mathscr{M}_{i}\right)\right)(i=$ $1,2, \ldots, m, j=1,2, \ldots, n)$ be the collection of q-ROFS RNs. $^{-}$ Let the weight vectors $k=\left(k_{1}, k_{2}, \ldots, k_{m}\right)^{T}$ and $l=\left(l_{1}\right.$, $\left.l_{2}, \ldots, l_{n}\right)^{T}$ for experts $s_{i}$ and parameters $c_{j}$ with $\sum_{i=1}^{m} t_{i}=1$, $\sum_{j=1}^{n} v_{j}=1$, and $0 \leq k_{i}, l_{j} \leq 1$. Consider $t=\left(t_{1}, t_{2}, \ldots\right.$, $\left.t_{m}\right)^{T}$ and $v=\left(v_{1}, v_{2}, \ldots, v_{n}\right)^{T}$ as the associated weight vectors of experts $s_{i}$ and parameters $c_{j}$ with $\sum_{i=1}^{m} t_{i}=1, \sum_{j=1}^{n} v_{j}=1$, and $0 \leq t_{i}, v_{j} \leq 1$, respectively. The $q-$ ROFS $_{t} R H A$ operator is defined as

$$
\mathrm{q}-\operatorname{ROFS}_{t} \operatorname{RHA}\left(\mathscr{L}_{c_{1}}\left(\mathscr{M}_{1}\right), \ldots, \mathscr{L}_{c_{n}}\left(\mathscr{M}_{m}\right)\right)=\left(\oplus_{j=1}^{n} v_{j}\left(\oplus_{i=1}^{m} t_{i} \underline{\mathscr{L}_{\delta c_{j}}^{*}}\left(\mathscr{M}_{i}\right)\right), \oplus_{j=1}^{n} v_{j}\left(\oplus_{i=1}^{m} t_{i} \overline{\mathscr{L}_{\delta c_{j}}^{*}}\left(\mathscr{M}_{i}\right)\right)\right) .
$$

From the above definition, the aggregated result for

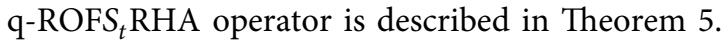

Theorem 5. Let $\mathscr{L}_{c_{j}}\left(\mathscr{M}_{i}\right)=\left(\mathscr{L}_{c_{j}}\left(\mathscr{M}_{i}\right), \overline{\mathscr{L}_{c_{j}}}\left(\mathscr{M}_{i}\right)\right)(i=$ $1,2, \ldots, m, j=1,2, \ldots, n)$ be the collection of $q-$ ROFS $_{t} R N s$. Let the weight vectors $k=\left(k_{1}, k_{2}, \ldots, k_{m}\right)^{T}$ and $l=\left(l_{1}, l_{2}\right.$, $\left.\ldots, l_{n}\right)^{T}$ of experts $s_{i}$ and parameters $c_{j}$ with $\sum_{i=1}^{m} t_{i}=1$, $\sum_{j=1}^{n} v_{j}=1$, and $0 \leq k_{i}, l_{j} \leq 1$. Consider the associated weight vectors $t=\left(t_{1}, t_{2}, \ldots, t_{m}\right)^{T}$ and $v=\left(v_{1}, v_{2}, \ldots, v_{n}\right)^{T}$ of experts $s_{i}$ and parameters $c_{j}$ with $\sum_{i=1}^{m} t_{i}=1, \sum_{j=1}^{n} v_{j}=1$, and $0 \leq t_{i}, v_{j} \leq 1$, respectively. Then, $q-R_{0 F S} R H A$ operator is given as

$$
\begin{aligned}
& \mathrm{q}-\operatorname{ROFS}_{t} \operatorname{RHA}\left(\mathscr{L}_{c_{1}}\left(\mathscr{M}_{1}\right), \ldots, \mathscr{L}_{c_{n}}\left(\mathscr{M}_{m}\right)\right)=\left(\oplus_{j=1}^{n} v_{j}\left(\oplus_{i=1}^{m} t_{i} \mathscr{L}_{\delta c_{j}}^{*}\left(\mathscr{M}_{i}\right)\right), \oplus_{j=1}^{n} v_{j}\left(\oplus_{i=1}^{m} t_{i} \overline{\mathscr{L}_{\delta c_{j}}^{*}}\left(\mathscr{M}_{i}\right)\right)\right)
\end{aligned}
$$

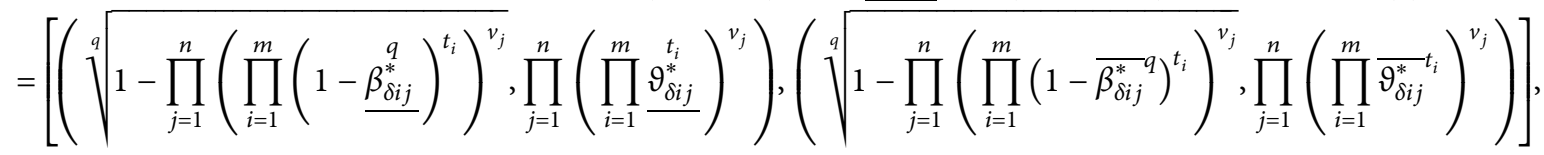

where $\mathscr{L}_{\delta c_{j}}^{*}\left(\mathscr{M}_{i}\right)=n k l \mathscr{L}_{c_{j}}=\left(n k l \mathscr{L}_{c_{j}}\left(\mathscr{M}_{i}\right), n k l \overline{\mathscr{L}_{c_{j}}}\left(\mathscr{M}_{i}\right)\right)$ denotes the largest value of the permutation from ith row and jth column of the collection $i \times j \quad q-R O F S_{t} R N s \quad \mathscr{L}_{c_{j}}\left(\mathscr{M}_{i}\right)=$ $\left(\mathscr{L}_{c_{j}}\left(\mathscr{M}_{i}\right), \overline{\mathscr{L}_{c_{j}}}\left(\mathscr{M}_{i}\right)\right)$ and $n$ represents the balancing coefficient. 
Example 4. Consider Table 3 of Example 2, for the collection q-ROFS RNs $\quad \mathscr{L}_{c_{j}}\left(\mathscr{M}_{i}\right)=\left(\mathscr{L}_{c_{j}}\left(\mathscr{M}_{i}\right), \overline{\mathscr{L}_{c_{j}}}\left(\mathscr{M}_{i}\right)\right) \quad$ with $k=(0.33,0.37,0.3)^{T}$ and $l=(\overline{0.42}, 0.58)^{T}$ as the weight vectors of experts $s_{i}$ and parameters $c_{j}$. Consider
$t=(0.36,0.34,0.3)^{T}$ and $v=(0.55,0.45)^{T}$ as the associated weight vectors of experts $s_{i}$ and parameters $c_{j}$. The tabular representation of $\mathscr{L}_{\delta c_{j}}^{*}\left(\mathscr{M}_{i}\right)$ through operation law and score function is given in Tables 5 and 6. Now,

$$
\begin{aligned}
& \mathrm{q}-\operatorname{ROFS}_{t} \operatorname{RHA}\left(\mathscr{L}_{c_{1}}\left(\mathscr{M}_{1}\right), \ldots, \mathscr{L}_{c_{n}}\left(\mathscr{M}_{m}\right)\right)=\left(\oplus_{j=1}^{2} v_{j}\left(\oplus_{i=1}^{3} t_{i} \underline{\mathscr{L}_{\delta c_{j}}^{*}}\left(\mathscr{M}_{i}\right)\right), \oplus_{j=1}^{2} v_{j}\left(\oplus_{i=1}^{3} t_{i} \overline{\mathscr{L}_{\delta c_{j}}^{*}}\left(\mathscr{M}_{i}\right)\right)\right), \\
& \mathrm{q}-\operatorname{ROFS}_{t} \operatorname{RHA}\left(\mathscr{L}_{c_{1}}\left(\mathscr{M}_{1}\right), \ldots, \mathscr{L}_{c_{n}}\left(\mathscr{M}_{m}\right)\right)=[(0.701609,0.129765),(0.600425,0.122969)] .
\end{aligned}
$$

From the analysis of Theorem 5, the following characteristics hold in $\mathrm{q}-\mathrm{ROFS}_{t} \mathrm{RHA}$ operator.

Theorem 6. Let $\mathscr{L}_{c_{j}}\left(\mathscr{M}_{i}\right)=\left(\mathscr{L}_{c_{j}}\left(\mathscr{M}_{i}\right), \overline{\mathscr{L}_{c_{j}}}\left(\mathscr{M}_{i}\right)\right)(i=1,2$, $\ldots, m, j=1,2, \ldots, n)$ be the collection of $q-R_{0 F S} R N s$. Let the weight vectors $k=\left(k_{1}, k_{2}, \ldots, k_{m}\right)^{T}$ and $l=\left(l_{1}\right.$, $\left.l_{2}, \ldots, l_{n}\right)^{T}$ of experts $s_{i}$ and parameters $c_{j}$ with $\sum_{i=1}^{m} t_{i}=1$, $\sum_{j=1}^{n} v_{j}=1$, and $0 \leq k_{i}, l_{j} \leq 1$. Consider the associated weight vectors $t=\left(t_{1}, t_{2}, \ldots, t_{m}\right)^{T}$ and $v=\left(v_{1}, v_{2}, \ldots, v_{n}\right)^{T}$ of experts $s_{i}$ and parameters $c_{j}$ with $\sum_{i=1}^{m} t_{i}=1, \sum_{j=1}^{n} v_{j}=1$, and $0 \leq t_{i}, v_{j} \leq 1$, respectively. Then, the following properties hold

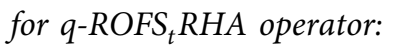

(i) (Idempotency): if $\mathscr{L}_{\delta c_{j}}^{*}\left(\mathscr{M}_{i}\right)=\mathscr{P}_{c}(\mathscr{N}) \quad($ for all $i=$ $1,2, \ldots$, m and $j=1,2, \ldots, n)$ where $\mathscr{P}_{c}(\mathscr{M})=\left(\mathscr{P}_{c}\right.$ $\left.(\mathscr{M}), \overline{\mathscr{P}}_{c}(\mathscr{M})\right)=((\underline{b}, \underline{d}),(\bar{b}, \bar{d}))$, then

$\mathrm{q}-\operatorname{ROFS}_{t} \operatorname{RHA}\left(\mathscr{L}_{c_{1}}\left(\mathscr{M}_{1}\right), \mathscr{L}_{c_{2}}\left(\mathscr{M}_{2}\right), \ldots, \mathscr{L}_{c_{n}}\left(\mathscr{M}_{m}\right)\right)=\mathscr{P}_{c}(\mathscr{M})$.

(ii) (Boundedness): let $\left(\mathscr{L}_{\delta c_{j}}^{*}\left(\mathscr{M}_{i}\right)\right)^{-}=\left(\min _{j} \min _{i}\right.$ $\left.\underline{\mathscr{L}_{\delta c_{j}}^{*}}\left(\mathscr{M}_{i}\right), \max _{j} \max _{i} \overline{\mathscr{L}_{\delta c_{j}}^{*}}\left(\mathscr{M}_{i}\right)\right)$ and $\left(\mathscr{L}_{\delta c_{j}}^{*}\left(\mathscr{M}_{i}\right)\right)^{+}$ $=\left(\max _{j} \max _{i} \quad \mathscr{L}_{\delta c_{j}}^{*}\left(\mathscr{M}_{i}\right), \min _{j} \min _{i} \overline{\mathscr{L}_{\delta c_{j}}^{*}}\left(\mathscr{M}_{i}\right)\right)$.

Then,

$$
\begin{aligned}
& \left(\mathscr{L}_{\delta c_{j}}^{*}\left(\mathscr{M}_{i}\right)\right)^{-} \leq \mathrm{q}-\operatorname{ROFS}_{t} \operatorname{RHA}\left(\mathscr{L}_{c_{1}}\left(\mathscr{M}_{1}\right), \mathscr{L}_{c_{2}}\left(\mathscr{M}_{2}\right), \ldots,\right. \\
& \left.\mathscr{L}_{c_{n}}\left(\mathscr{M}_{m}\right)\right) \leq\left(\mathscr{L}_{\delta c_{j}}^{*}\left(\mathscr{M}_{i}\right)\right)^{+} .
\end{aligned}
$$

(iii) (Monotonicity): let $\mathscr{P}_{c_{j}}\left(\mathscr{N}_{i}\right)=\left(\mathscr{P}_{c_{j}}\left(\mathscr{N}_{i}\right), \overline{\mathscr{P}_{c_{j}}}\right.$ $\left.\left(\mathcal{N}_{i}\right)\right)(i=1,2, \ldots, m, j=1,2, \ldots, n) \overline{\text { be }}$ another $\mathscr{\mathscr { P }}_{c_{j}}^{\text {collection }}\left(\mathscr{N}_{i}\right) \leq \mathscr{L}_{c_{j}}^{\text {of }}\left(\mathscr{M}_{i}\right)^{q-R O F S_{t} R N s}$ and $\overline{\mathscr{P}}_{c_{j}}\left(\mathscr{N}_{i}\right) \leq \overline{\mathscr{L}}\left(\mathscr{M}_{i}\right)$. Then,

$$
\begin{aligned}
\mathrm{q} & -\operatorname{ROFS}_{t} \operatorname{RHA}\left(\mathscr{P}_{c_{1}}\left(\mathscr{M}_{1}\right), \mathscr{P}_{c_{2}}\left(\mathscr{M}_{2}\right), \ldots, \mathscr{P}_{c_{n}}\left(\mathscr{M}_{m}\right)\right) \\
& \leq \mathrm{q}-\operatorname{ROFS}_{t} \operatorname{RHA}\left(\mathscr{L}_{c_{1}}\left(\mathscr{M}_{1}\right), \mathscr{L}_{c_{2}}\left(\mathscr{M}_{2}\right), \ldots, \mathscr{L}_{c_{n}}\left(\mathscr{M}_{m}\right)\right) .
\end{aligned}
$$

(iv) (Shift invariance): let $\mathscr{P}_{c}(\mathcal{N})=\left(\underline{\mathscr{P}_{c}}(\mathcal{N})\right.$, $\left.\overline{\mathscr{P}}_{c}(\mathcal{N})\right)=((\underline{b}, \underline{d}),(\bar{b}, \bar{d})) \quad$ be any ${ }^{c}$ other $q-\mathrm{ROFS}_{t} R N$. Then,

$$
\begin{aligned}
& \mathrm{q}- \operatorname{ROFS}_{t} \operatorname{RHA}\left(\mathscr{L}_{c_{1}}\left(\mathscr{M}_{1}\right) \oplus \mathscr{P}_{c}(\mathcal{N}), \mathscr{L}_{c_{2}}\left(\mathscr{M}_{2}\right)\right. \\
&\left.\oplus \mathscr{P}_{c}(\mathcal{N}), \ldots, \mathscr{L}_{c_{n}}\left(\mathscr{M}_{m}\right) \oplus \mathscr{P}_{c}(\mathcal{N})\right)=\mathrm{q}-\operatorname{ROFS}_{t} \operatorname{RHA} \\
&\left(\mathscr{L}_{c_{1}}\left(\mathscr{M}_{1}\right), \mathscr{L}_{c_{2}}\left(\mathscr{M}_{2}\right), \ldots, \mathscr{L}_{c_{n}}\left(\mathscr{M}_{m}\right)\right) \oplus \mathscr{P}_{c}(\mathcal{N}) .
\end{aligned}
$$

(v) (Homogeneity): for any real number $\lambda>0$,

$$
\begin{aligned}
\mathrm{q} & -\operatorname{ROFS}_{t} \operatorname{RHA}\left(\lambda \mathscr{L}_{c_{1}}\left(\mathscr{M}_{1}\right), \lambda \mathscr{L}_{c_{2}}\left(\mathscr{M}_{2}\right), \ldots, \lambda \mathscr{L}_{c_{n}}\left(\mathscr{M}_{m}\right)\right) \\
& =\lambda \mathrm{q}-\operatorname{ROFS} \operatorname{RHA}_{t}\left(\mathscr{L}_{c_{1}}\left(\mathscr{M}_{1}\right), \mathscr{L}_{c_{2}}\left(\mathscr{M}_{2}\right), \ldots, \mathscr{L}_{c_{n}}\left(\mathscr{M}_{m}\right)\right) .
\end{aligned}
$$

Remark 4

(a) If $q=1$, then $\mathrm{q}-\mathrm{ROFS}_{t} \mathrm{RHA}$ operator reduces to $\mathrm{IFS}_{t}$ RHA operator

(b) If $q=2$, then $\mathrm{q}-\mathrm{ROFS}_{t} \mathrm{RHA}$ operator reduces to $\mathrm{PFS}_{t}$ RHA operator

(c) If soft parameter $c_{1}$ is one (means $n=1$ ), then $\mathrm{q}-\mathrm{ROFS}_{t} \mathrm{RHA}$ operator reduces to q-ROFRHA operator

(d) If $k l=((1 / n),(1 / n), \ldots,(1 / n))^{T}$, then the proposed $\mathrm{q}-\mathrm{ROFS}_{t} \mathrm{RHA}$ operator reduces to q-ROFS $\mathrm{RWA}^{-}$ operator

(e) If $t v=((1 / n),(1 / n), \ldots,(1 / n))^{T}$, then the proposed q-ROFS RHA operator reduces to q-ROFS $_{t}$ ROWA operator

\section{5. q-Rung Orthopair Fuzzy Soft Rough \\ Geometric (q-ROFS $S_{t} \mathbf{R G )}$ Aggregation Operator}

This section is devoted to the study of $\mathrm{q}-\mathrm{ROFS}_{t} \mathrm{R}$ geometric

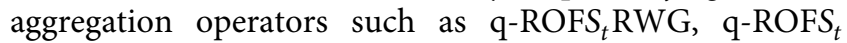
ROWG, and q-ROFS $S_{t}$ RHG operators. We will present the fundamental properties of these operators in detail. 
TABLE 5: Tabular representation by using operation law for $\mathscr{L}_{\delta c_{j}}^{*}\left(\mathscr{M}_{i}\right)=n k l \mathscr{L}_{c_{j}}=\left(n k l \underline{\mathscr{L}_{c_{j}}}\left(\mathscr{M}_{i}\right), n k l \overline{\mathscr{L}_{c_{j}}}\left(\mathscr{M}_{i}\right)\right)$.

\begin{tabular}{lcc}
\hline $\mathscr{L}$ & $c_{1}$ & $c_{2}$ \\
\hline$s_{1}$ & $((0.7483,0.1247),(0.6366,0.1663))$ & $((0.4629,0.1148),(0.6561,0.0804))$ \\
$s_{2}$ & $((0.5624,0.0466),(0.1551,0.3497))$ & $((0.8533,0.1931),(0.5254,0.1931))$ \\
$s_{3}$ & $((0.7574,0.0945),(0.4853,0.0567))$ & $((0.3238,0.4437),(0.7661,0.0626))$ \\
\hline
\end{tabular}

TABLE 6: Tabular representation after using score function $\mathscr{L}_{\delta c_{j}}^{*}\left(\mathscr{M}_{i}\right)=\left(\mathscr{L}_{\delta c_{j}}^{*}\left(\mathscr{M}_{i}\right), \overline{\mathscr{L}_{\delta c_{j}}^{*}}\left(\mathscr{M}_{i}\right)\right)$.

\begin{tabular}{lcc}
\hline $\mathscr{L}$ & $c_{1}$ & $c_{2}$ \\
\hline$s_{1}$ & $((0.7483,0.1247),(0.6366,0.1663))$ & $((0.8533,0.1931),(0.5254,0.1931))$ \\
$s_{2}$ & $((0.7574,0.0945),(0.4853,0.0567))$ & $((0.3238,0.4437),(0.7661,0.0626))$ \\
$s_{3}$ & $((0.5624,0.0466),(0.1551,0.3497))$ & $((0.4629,0.1148),(0.6561,0.0804))$ \\
\hline
\end{tabular}

5.1. q-Rung Orthopair Fuzzy Soft Rough Weighted Geometric $\left(q-R^{2} \mathrm{FS}_{t} R W G\right)$ Operator

Definition 14. Let $\mathscr{L}_{c_{j}}\left(\mathscr{M}_{i}\right)=\left(\mathscr{L}_{c_{j}}\left(\mathscr{M}_{i}\right), \overline{\mathscr{L}_{c_{j}}}\left(\mathscr{M}_{i}\right)\right)(i=$ $1,2, \ldots, m, j=1,2, \ldots, n)$ be the collection of $\mathrm{q}-\mathrm{ROFS}_{t} \mathrm{RNs}$.
Let the weight vectors $t=\left(t_{1}, t_{2}, \ldots, t_{m}\right)^{T}$ and $v=\left(v_{1}, v_{2}, \ldots, v_{n}\right)^{T}$ of experts $s_{i}$ and parameters $c_{j}$ with $\sum_{i=1}^{m} t_{i}=1, \sum_{j=1}^{n} v_{j}=1$, and $0 \leq t_{i}, v_{j} \leq 1$, respectively. The q-ROFS ${ }_{t}$ RWG operator is defined as

$$
\mathrm{q}-\operatorname{ROFS}_{t} \operatorname{RWG}\left(\mathscr{L}_{c_{1}}\left(\mathscr{M}_{1}\right), \ldots, \mathscr{L}_{c_{n}}\left(\mathscr{M}_{m}\right)\right)=\left[\oplus_{j=1}^{n}\left\{\oplus_{i=1}^{m}\left(\underline{\mathscr{L}_{c_{j}}}\left(\mathscr{M}_{i}\right)\right)^{t_{i}}\right\}^{v_{j}}, \oplus_{j=1}^{n}\left\{\oplus_{i=1}^{m}\left(\overline{\mathscr{L}_{c_{j}}}\left(\mathscr{M}_{i}\right)\right)^{t_{i}}\right\}^{v_{j}}\right]
$$

Based on the above definition the aggregated result for $\mathrm{q}-\mathrm{ROFS}_{t} \mathrm{RWG}$ operator is given in Theorem 7 .

Theorem 7. Let $\quad \mathscr{L}_{c_{j}}\left(\mathscr{M}_{i}\right)=\left(\mathscr{L}_{c_{j}}\left(\mathscr{M}_{i}\right), \overline{\mathscr{L}_{c_{j}}}\left(\mathscr{M}_{i}\right)\right)$ $(i=1,2, \ldots, m, j=1,2, \ldots, n)$ be the collection of
q-ROFS ${ }_{t} R N s$ Let the weight vectors $t=\left(t_{1}, t_{2}, \ldots, t_{m}\right)^{T}$ and $v=\left(v_{1}, v_{2}, \ldots, v_{n}\right)^{T}$ of experts $s_{i}$ and parameters $c_{j}$ with $\sum_{i=1}^{m} t_{i}=1, \sum_{j=1}^{n} v_{j}=1$, and $0 \leq t_{i}, v_{j} \leq 1$, respectively. Then, $q-R^{2} O F S_{t} R W G$ operator is given as

$$
\begin{aligned}
& \mathrm{q}-\operatorname{ROFS}_{t} \operatorname{RWG}\left(\mathscr{L}_{c_{1}}\left(\mathscr{M}_{1}\right), \ldots, \mathscr{L}_{c_{n}}\left(\mathscr{M}_{m}\right)\right)=\left[\oplus_{j=1}^{n}\left\{\oplus_{i=1}^{m}\left(\underline{\mathscr{L}_{c_{j}}}\left(\mathscr{M}_{i}\right)\right)^{t_{i}}\right\}^{v_{j}}, \oplus_{j=1}^{n}\left\{\oplus_{i=1}^{m}\left(\overline{\mathscr{L}_{c_{j}}}\left(\mathscr{M}_{i}\right)\right)^{t_{i}}\right\}^{v_{j}}\right] \\
& =\left[\left\{\prod_{j=1}^{n}\left(\prod_{i=1}^{m} \frac{\beta_{i j}}{t_{i}}\right)^{v_{j}}, \sqrt[q]{1-\prod_{j=1}^{n}\left(\prod_{i=1}^{m}\left(1-\underline{\vartheta_{i j}^{q}}\right)^{t_{i}}\right)^{v_{j}}}\right\},\left\{\prod_{j=1}^{n}\left(\prod_{i=1}^{m} \overline{\beta_{i j}} t^{t_{i}}\right)^{v_{j}}, \sqrt[q]{\left.1-\prod_{j=1}^{n}\left(\prod_{i=1}^{m}\left(1-\overline{\vartheta_{i j}}\right)^{t_{i}}\right)^{v_{j}}\right\}} .\right.\right.
\end{aligned}
$$

Since it is clear that $\mathscr{L}_{c_{j}}\left(\mathscr{M}_{i}\right)$ and $\overline{\mathscr{L}_{c_{j}}}\left(\mathscr{M}_{i}\right)$ are q-ROFNs, by Definition 7 , we have that

$$
\begin{aligned}
& \oplus_{j=1}^{n}\left\{\oplus_{i=1}^{m}\left(\underline{\mathscr{L}_{c_{j}}}\left(\mathscr{M}_{i}\right)\right)^{t_{i}}\right\}^{v_{j}} \text { and } \oplus_{j=1}^{n}\left\{\oplus_{i=1}^{m}\left(\overline{\mathscr{L}_{c_{j}}}\left(\mathscr{M}_{i}\right)\right)^{t_{i}}\right\}^{v_{j}} \\
& \text { are also q-ROFNs. Therefore, }
\end{aligned}
$$


$\mathrm{q}-\operatorname{ROFS}_{t} \operatorname{RWG}\left(\mathscr{L}_{c_{1}}\left(\mathscr{M}_{1}\right), \ldots, \mathscr{L}_{c_{n}}\left(\mathscr{M}_{m}\right)\right)$ is also a q-ROFS ${ }_{t} \mathrm{RN}$ in approximation space $(\mathscr{T}, \mathscr{E}, \mathscr{L})$.
Example 5. Consider Table 3 of Example 2, for the collection q-ROFS $S_{t}$ RNs $\mathscr{L}_{c_{j}}\left(\mathscr{M}_{i}\right)=\left(\mathscr{L}_{c_{j}}\left(\mathscr{M}_{i}\right), \overline{\mathscr{L}_{c_{j}}}\left(\mathscr{M}_{i}\right)\right)$. Then, the aggregated result for $\mathrm{q}-\mathrm{R} \overline{\mathrm{OFS}}_{t} \mathrm{RWG}$ is given as

$$
\begin{aligned}
\mathrm{q} & =\operatorname{ROFS}_{t} \operatorname{RWG}\left(\mathscr{L}_{c_{1}}\left(\mathscr{M}_{1}\right), \ldots, \mathscr{L}_{c_{n}}\left(\mathscr{M}_{m}\right)\right)=\left[\oplus_{j=1}^{2}\left\{\oplus_{i=1}^{3}\left(\underline{\mathscr{L}_{c_{j}}}\left(\mathscr{M}_{i}\right)\right)^{t_{i}}\right\}^{v_{j}}, \oplus_{j=1}^{2}\left\{\oplus_{i=1}^{3}\left(\overline{\mathscr{L}}_{c_{j}}\left(\mathscr{M}_{i}\right)\right)^{t_{i}}\right\}^{v_{j}}\right] \\
= & {\left[\left\{\left(0.9^{0.25} 0.7^{0.3} 0.92^{0.45}\right)^{0.55}\left(0.55^{0.25} 0.92^{0.3} 0.4^{0.45}\right)^{0.45},\right.\right.} \\
& \sqrt{3} 1-\left(\left[\left(1-0.3^{3}\right)^{0.25}\left(1-0.1^{3}\right)^{0.25}\left(1-0.25^{3}\right)^{0.45}\right]^{0.55}\left[\left(1-0.2^{3}\right)^{0.25}\left(1-0.3^{3}\right)^{0.25}\left(1-0.85^{3}\right)^{0.45}\right]\right\}, \\
& \left\{\left(0.8^{0.25} 0.2^{0.3} 0.65^{0.45}\right)^{0.55}\left(0.76^{0.25} 0.6^{0.3} 0.88^{0.45}\right)^{0.45},\right. \\
& \left.\left.\sqrt{3} 1-\left(\left[\left(1-0.4^{3}\right)^{0.25}\left(1-0.75^{3}\right)^{0.25}\left(1-0.15^{3}\right)^{0.45}\right]^{0.55}\left[\left(1-0.14^{3}\right)^{0.25}\left(1-0.3^{3}\right)^{0.25}\left(1-0.12^{3}\right)^{0.45}\right]^{0.45}\right)\right\}\right] \\
= & {[(0.715607,0.509925),(0.573442,0.484819)] . }
\end{aligned}
$$

From the analysis of Theorem 7, the following hold for q-ROFS ${ }_{t}$ RWG operator.

Theorem 8. Let $\quad \mathscr{L}_{c_{j}}\left(\mathscr{M}_{i}\right)=\left(\mathscr{L}_{c_{j}}\left(\mathscr{M}_{i}\right), \overline{\mathscr{L}_{c_{j}}}\left(\mathscr{M}_{i}\right)\right)(i=$ $1,2, \ldots, m, j=1,2, \ldots, n)$ be the collection of $q-R^{2} O F S_{t} R N s$. Let the weight vectors $t=\left(t_{1}, t_{2}, \ldots, t_{m}\right)^{T}$ and $v=\left(v_{1}\right.$, $\left.v_{2}, \ldots, v_{n}\right)^{T}$ of experts $s_{i}$ and parameters $c_{j}$ with $\sum_{i=1}^{m} t_{i}=1$, $\sum_{j=1}^{n} v_{j}=1$, and $0 \leq t_{i}, v_{j} \leq 1$, respectively. Then, the following properties hold for $q$-ROFS $S_{t} R W G$ operator:

(i) (Idempotency): if $\mathscr{L}_{c_{j}}\left(\mathscr{M}_{i}\right)=\mathscr{P}_{c}(\mathcal{N}) \quad$ (for all $i=$ $1,2, \ldots$, mand $j=1,2, \ldots, n)$ where $\mathscr{P}_{c}(\mathscr{M})=$ $\left(\mathscr{P}_{c}(\mathscr{M}), \overline{\mathscr{P}}_{c}(\mathscr{M})\right)=((\underline{b}, \underline{d}),(\bar{b}, \bar{d}))$, then

$$
\begin{aligned}
\mathrm{q} & -\operatorname{ROFS}_{t} \operatorname{RWG}\left(\mathscr{L}_{c_{1}}\left(\mathscr{M}_{1}\right), \mathscr{L}_{c_{2}}\left(\mathscr{M}_{2}\right), \ldots, \mathscr{L}_{c_{n}}\left(\mathscr{M}_{m}\right)\right) \\
& =\mathscr{P}_{c}(\mathscr{M}) .
\end{aligned}
$$

(ii) (Boundedness): let $\left(\mathscr{L}_{c_{j}}\left(\mathscr{M}_{i}\right)\right)^{-}=\left(\min _{j} \min _{i} \mathscr{L}_{c_{j}}\right.$ $\left.\left(\mathscr{M}_{i}\right), \max _{j} \max _{i} \overline{\mathscr{L}_{c_{j}}}\left(\mathscr{M}_{i}\right)\right)$ and $\left(\mathscr{L}_{c_{j}}\left(\mathscr{M}_{i}\right)\right)^{+}=$ $\left(\max _{j} \max _{i} \mathscr{L}_{c_{j}}\left(\mathscr{M}_{i}\right), \min _{j} \min _{i} \overline{\mathscr{L}}_{c_{j}}\left(\mathscr{M}_{i}\right)\right)$. Then,

$$
\begin{aligned}
\left(\mathscr{L}_{c_{j}}\left(\mathscr{M}_{i}\right)\right)^{-} & \leq \mathrm{q}-\operatorname{ROFS}_{t} \operatorname{RWG}\left(\mathscr{L}_{c_{1}}\left(\mathscr{M}_{1}\right), \mathscr{L}_{c_{2}}\left(\mathscr{M}_{2}\right), \ldots,\right. \\
\left.\mathscr{L}_{c_{n}}\left(\mathscr{M}_{m}\right)\right) & \leq\left(\mathscr{L}_{c_{j}}\left(\mathscr{M}_{i}\right)\right)^{+}
\end{aligned}
$$

(iii) (Monotonicity): let $\quad \mathscr{P}_{c_{j}}\left(\mathcal{N}_{i}\right)=\left(\mathscr{P}_{c_{j}}\left(\mathscr{N}_{i}\right), \overline{\mathscr{P}_{c_{j}}}\right.$ $\left.\left(\mathcal{N}_{i}\right)\right)(i=1,2, \ldots, m, j=1,2, \ldots, n)$ be another collection of $q-R O F S_{t} R N s$ such that $\mathscr{P}_{c_{j}}\left(\mathcal{N}_{i}\right) \leq$ $\mathscr{L}_{c_{j}}\left(\mathscr{M}_{i}\right)$ and $\overline{\mathscr{P}_{c_{j}}}\left(\mathscr{N}_{i}\right) \leq \overline{\mathscr{L}_{c_{j}}}\left(\mathscr{M}_{i}\right)$. Then,

$$
\begin{aligned}
\mathrm{q}- & \operatorname{ROFS}_{t} \operatorname{RWG}\left(\mathscr{P}_{c_{1}}\left(\mathscr{M}_{1}\right), \mathscr{P}_{c_{2}}\left(\mathscr{M}_{2}\right), \ldots, \mathscr{P}_{c_{n}}\left(\mathscr{M}_{m}\right)\right) \\
& \leq \mathrm{q}-\operatorname{ROFS}_{t} \operatorname{RWG}\left(\mathscr{L}_{c_{1}}\left(\mathscr{M}_{1}\right), \mathscr{L}_{c_{2}}\left(\mathscr{M}_{2}\right), \ldots, \mathscr{L}_{c_{n}}\left(\mathscr{M}_{m}\right)\right) .
\end{aligned}
$$

(iv) (Shift invariance): let $\mathscr{P}_{c}(\mathcal{N})=\left(\mathscr{P}_{c}(\mathcal{N}), \overline{\mathscr{P}_{c}}\right.$ $(\mathcal{N}))=((\underline{b}, \underline{d}),(\bar{b}, \bar{d}))$ be any other $q-\bar{c} O F S_{t} R N$. Then,

$$
\begin{aligned}
\mathrm{q}- & \operatorname{ROFS}_{t} \operatorname{RWG}\left(\mathscr{L}_{c_{1}}\left(\mathscr{M}_{1}\right) \oplus \mathscr{P}_{c}(\mathcal{N}), \mathscr{L}_{c_{2}}\left(\mathscr{M}_{2}\right)\right. \\
& \left.\oplus \mathscr{P}_{c}(\mathcal{N}), \ldots, \mathscr{L}_{c_{n}}\left(\mathscr{M}_{m}\right) \oplus \mathscr{P}_{c}(\mathscr{N})\right) \\
=\mathrm{q}- & \operatorname{ROFS}_{t} \operatorname{RWG}\left(\mathscr{L}_{c_{1}}\left(\mathscr{M}_{1}\right), \mathscr{L}_{c_{2}}\left(\mathscr{M}_{2}\right), \ldots, \mathscr{L}_{c_{n}}\left(\mathscr{M}_{m}\right)\right) \\
& \oplus \mathscr{P}_{c}(\mathscr{N}) .
\end{aligned}
$$

(v) (Homogeneity): for any real number $\lambda>0$,

$$
\begin{aligned}
\mathrm{q} & -\operatorname{ROFS}_{t} \operatorname{RWG}\left(\lambda \mathscr{L}_{c_{1}}\left(\mathscr{M}_{1}\right), \lambda \mathscr{L}_{c_{2}}\left(\mathscr{M}_{2}\right), \ldots, \lambda \mathscr{L}_{c_{n}}\left(\mathscr{M}_{m}\right)\right) \\
\quad & =\lambda \mathrm{q}-\operatorname{ROF} S_{t} \operatorname{RWG}\left(\mathscr{L}_{c_{1}}\left(\mathscr{M}_{1}\right), \mathscr{L}_{c_{2}}\left(\mathscr{M}_{2}\right), \ldots, \mathscr{L}_{c_{n}}\left(\mathscr{M}_{m}\right)\right) .
\end{aligned}
$$

Remark 5

(a) If $q=1$, then $\mathrm{q}-\mathrm{ROFS}_{t} \mathrm{RWG}$ operator reduces to $\mathrm{IFS}_{t} \mathrm{RWG}$ operator

(b) If $q=2$, then $\mathrm{q}-\mathrm{ROFS}_{t} \mathrm{RWG}$ operator reduces to $\mathrm{PFS}_{t}$ RWG operator

(c) If soft parameter $c_{1}$ is one (means $\left.n=1\right)$, then $\mathrm{q}-\mathrm{ROFS}_{t} \mathrm{RWG}$ operator reduces to q-ROFRWG operator 
5.2. q-Rung Orthopair Fuzzy Soft Rough Ordered Weighted Geometric ( $q$-ROFS ROWG $_{t}$ Operator. Here, we will put forward the detailed study of q-ROFS $\mathrm{ROWG}_{t}$ operator and some of its fundamental properties. The q-ROFS $\mathrm{ROWG}^{\mathrm{RO}}$ operator weights the ordered position of the q-ROFVs instead of weighting the values themselves.
Definition 15. Let $\quad \mathscr{L}_{c_{j}}\left(\mathscr{M}_{i}\right)=\left(\mathscr{L}_{c_{j}}\left(\mathscr{M}_{i}\right), \overline{\mathscr{L}_{c_{j}}}\left(\mathscr{M}_{i}\right)\right)$ $(i=1,2, \ldots, m, j=1,2, \ldots, n)$ be the collection of q-ROFS ${ }_{t}$ RNs. Let the weight vectors $t=\left(t_{1}\right.$, $\left.t_{2}, \ldots, t_{m}\right)^{T}$ and $v=\left(v_{1}, v_{2}, \ldots, v_{n}\right)^{T}$ of experts $s_{i}$ and parameters $c_{j}$ with $\sum_{i=1}^{m} t_{i}=1, \sum_{j=1}^{n} v_{j}=1$, and $0 \leq t_{i}, v_{j} \leq 1$, respectively. The $\mathrm{q}-\mathrm{ROFS}_{t} \mathrm{ROWG}$ operator is defined as

$$
\mathrm{q}-\operatorname{ROFS}_{t} \operatorname{ROWG}\left(\mathscr{L}_{c_{1}}\left(\mathscr{M}_{1}\right), \ldots, \mathscr{L}_{c_{n}}\left(\mathscr{M}_{m}\right)\right)=\left[\oplus_{j=1}^{n}\left\{\oplus_{i=1}^{m}\left(\underline{\mathscr{L}_{\delta c_{j}}}\left(\mathscr{M}_{i}\right)\right)^{t_{i}}\right\}^{v_{j}}, \oplus_{j=1}^{n}\left\{\oplus_{i=1}^{m}\left(\overline{\mathscr{L}_{\delta c_{j}}}\left(\mathscr{M}_{i}\right)\right)^{t_{i}}\right\}^{v_{j}}\right]
$$

In view of the above definition, the aggregated result for $\mathrm{q}-\mathrm{ROFS}_{t}$ ROWG operator is described in Theorem 9.

Theorem 9. Let $\quad \mathscr{L}_{c_{j}}\left(\mathscr{M}_{i}\right)=\left(\mathscr{L}_{c_{j}}\left(\mathscr{M}_{i}\right), \overline{\mathscr{L}_{c_{j}}}\left(\mathscr{M}_{i}\right)\right)$ $(i=1,2, \ldots, m, j=1,2, \ldots, n)$ be the collection of
q-ROFS $S_{t}$ Ns. Let the weight vectors $t=\left(t_{1}, t_{2}, \ldots, t_{m}\right)^{T}$ and $v=\left(v_{1}, v_{2}, \ldots, v_{n}\right)^{T}$ of experts $s_{i}$ and parameters $c_{j}$ with $\sum_{i=1}^{m} t_{i}=1, \sum_{j=1}^{n} v_{j}=1$, and $0 \leq t_{i}, v_{j} \leq 1$, respectively. Then, $q-R O F S_{t} R O W G$ operator is given as

$$
\begin{aligned}
& \mathrm{q}-\operatorname{ROFS}_{t} \operatorname{ROWG}\left(\mathscr{L}_{c_{1}}\left(\mathscr{M}_{1}\right), \ldots, \mathscr{L}_{c_{n}}\left(\mathscr{M}_{m}\right)\right)=\left[\oplus_{j=1}^{n}\left\{\oplus_{i=1}^{m}\left(\underline{\mathscr{L}_{\delta c_{j}}}\left(\mathscr{M}_{i}\right)\right)^{t_{i}}\right\}^{v_{j}}, \oplus_{j=1}^{n}\left\{\oplus_{i=1}^{m}\left(\overline{\mathscr{L}_{\delta c_{j}}}\left(\mathscr{M}_{i}\right)\right)^{t_{i}}\right\}^{v_{j}}\right] \\
& =\left[\left\{\prod_{j=1}^{n}\left(\prod_{i=1}^{m} \frac{\beta_{\delta i j}^{t_{i}}}{{ }^{\prime}}\right)^{v_{j}}, \sqrt[q]{1-\prod_{j=1}^{n}\left(\prod_{i=1}^{m}\left(1-\underline{\vartheta_{\delta i j}^{q}}\right)^{t_{i}}\right)^{v_{j}}}\right\},\left\{\prod_{j=1}^{n}\left(\prod_{i=1}^{m} \overline{\beta_{\delta i j}} t^{t_{i}}\right)^{v_{j}}, \sqrt[q]{1-\prod_{j=1}^{n}\left(\prod_{i=1}^{m}\left(1-\overline{\vartheta_{\delta i j}}\right)^{t_{i}}\right)^{v_{j}}}\right\},\right.
\end{aligned}
$$

where $\mathscr{L}_{\delta c_{j}}\left(\mathscr{M}_{i}\right)=\left(\mathscr{L}_{\delta c_{j}}\left(\mathscr{M}_{i}\right), \overline{\mathscr{L}_{\delta c_{j}}}\left(\mathscr{M}_{i}\right)\right)$ denotes the largest value of the permutation from ith row and jth column of the collection $i \times j \quad q-R^{2} O F S_{t} R N s$, $\left.\mathscr{L}_{c_{j}}\left(\mathscr{M}_{i}\right)=\underline{\left(\mathscr{L}_{c_{j}}\right.}\left(\mathscr{M}_{i}\right), \overline{\mathscr{L}_{c_{j}}}\left(\mathscr{M}_{i}\right)\right)$.

Remark 6

(a) If $q=1$, then $\mathrm{q}-\mathrm{ROFS}_{t}$ ROWG operator reduces to IFS $_{t}$ ROWG operator

(b) If $q=2$, then $\mathrm{q}-\mathrm{ROFS}_{t} \mathrm{ROWG}$ operator reduces to $\mathrm{PFS}_{t}$ ROWG operator

(c) If the soft parameter $c_{1}$ is one (means $n=1$ ), then

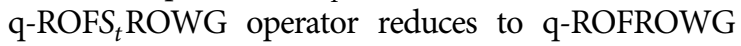
operator

5.3. q-Rung Orthopair Fuzzy Soft Rough Hybrid Geometric ( $q$ ROFS $_{t} R H G$ ) Operator. From the analysis of Definitions 14 and 15 , it is clear that the $\mathrm{q}-\mathrm{ROFS}_{t} \mathrm{RWG}$ operator weights

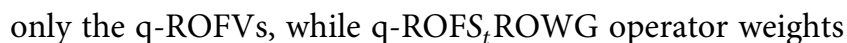
the ordered position of the q-ROFVs instead of weighting themselves. Motivated by the idea of combining weighted geometric and the ordered weighted geometric by using the combined notion of soft rough set, we present q-ROFS RHG $^{-}$ operator, which weights both the given q-ROFV and its ordered position. The basic desirable properties of the developed operator are presented in detail.

Definition 16. Let $\mathscr{L}_{c_{j}}\left(\mathscr{M}_{i}\right)=\left(\mathscr{L}_{c_{j}}\left(\mathscr{M}_{i}\right), \overline{\mathscr{L}_{c_{j}}}\left(\mathscr{M}_{i}\right)\right)(i=$ $1,2, \ldots, m, j=1,2, \ldots, n)$ be the collection of q-ROFS $\mathrm{RNs}^{\mathrm{R}}$. Let the weight vectors $k=\left(k_{1}, k_{2}, \ldots, k_{m}\right)^{T}$ and $l=\left(l_{1}\right.$, $\left.l_{2}, \ldots, l_{n}\right)^{T}$ of experts $s_{i}$ and parameters $c_{j}$ with $\sum_{i=1}^{m} t_{i}=1$, $\sum_{j=1}^{n} v_{j}=1$, and $0 \leq k_{i}, l_{j} \leq 1$. Consider the associated weight vectors $t=\left(t_{1}, t_{2}, \ldots, t_{m}\right)^{T}$ and $v=\left(v_{1}, v_{2}, \ldots, v_{n}\right)^{T}$ of experts $s_{i}$ and parameters $c_{j}$ with $\sum_{i=1}^{m} t_{i}=1, \sum_{j=1}^{n} v_{j}=1$, and $0 \leq t_{i}, v_{j} \leq 1$, respectively. The q-ROFS $\mathrm{RHG}$ operator is defined as

$$
\mathrm{q}-\operatorname{ROFS}_{t} \operatorname{RHG}\left(\mathscr{L}_{c_{1}}\left(\mathscr{M}_{1}\right), \ldots, \mathscr{L}_{c_{n}}\left(\mathscr{M}_{m}\right)\right)=\left[\oplus_{j=1}^{n}\left\{\oplus_{i=1}^{m}\left(\underline{\mathscr{L}_{\delta c_{j}}^{*}}\left(\mathscr{M}_{i}\right)\right)^{t_{i}}\right\}^{v_{j}}, \oplus_{j=1}^{n}\left\{\oplus_{i=1}^{m}\left(\overline{\mathscr{L}_{\delta c_{j}}^{*}}\left(\mathscr{M}_{i}\right)\right)^{t_{i}}\right\}^{v_{j}}\right] .
$$


From the above definition, the aggregated value for q-ROFS ${ }_{t}$ RHG operator is described in Theorem 11.

Theorem 10. Let $\mathscr{L}_{c_{j}}\left(\mathscr{M}_{i}\right)=\left(\mathscr{L}_{c_{j}}\left(\mathscr{M}_{i}\right), \overline{\mathscr{L}_{c_{j}}}\left(\mathscr{M}_{i}\right)\right)(i=$ $1,2, \ldots, m, j=1,2, \ldots, n)$ be the collection of $q-R^{2} \mathrm{FS}_{t} R N s$. Let the weight vectors $k=\left(k_{1}, k_{2}, \ldots, k_{m}\right)^{T}$ and $l=\left(l_{1}, l_{2}, \ldots, l_{n}\right)^{T}$ of experts $s_{i}$ and parameters $c_{j}$ with $\sum_{i=1}^{m} t_{i}=1, \sum_{j=1}^{n} v_{j}=1$, and $0 \leq k_{i}, l_{j} \leq 1$. Consider the associated weight vectors $t=\left(t_{1}, t_{2}, \ldots, t_{m}\right)^{T}$ and $v=$ $\left(v_{1}, v_{2}, \ldots, v_{n}\right)^{T}$ of experts $s_{i}$ and parameters $c_{j}$ with $\sum_{i=1}^{m} t_{i}=1, \sum_{j=1}^{n} v_{j}=1$, and $0 \leq t_{i}, v_{j} \leq 1$, respectively. Then,

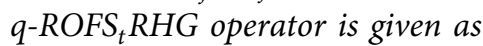

$$
\begin{aligned}
& \mathrm{q}-\operatorname{ROFS}_{t} \operatorname{RHG}\left(\mathscr{L}_{c_{1}}\left(\mathscr{M}_{1}\right), \ldots, \mathscr{L}_{c_{n}}\left(\mathscr{M}_{m}\right)\right)=\left[\oplus_{j=1}^{n}\left\{\oplus_{i=1}^{m}\left(\mathscr{L}_{\delta c_{j}}^{*}\left(\mathscr{M}_{i}\right)\right)^{t_{i}}\right\}^{v_{j}}, \oplus_{j=1}^{n}\left\{\oplus_{i=1}^{m}\left(\overline{\mathscr{L}_{\delta c_{j}}^{*}}\left(\mathscr{M}_{i}\right)\right)^{t_{i}}\right\}^{v_{j}}\right]
\end{aligned}
$$

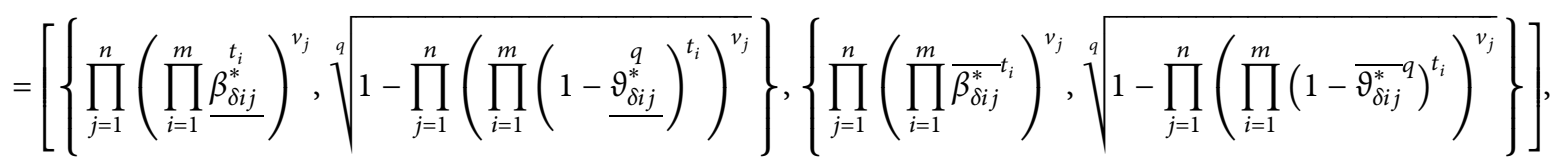

where $\mathscr{L}_{\delta c_{j}}^{*}\left(\mathscr{M}_{i}\right)=\left(\mathscr{L}_{c_{j}}\right)^{n k l}=\left(\left(\mathscr{L}_{c_{j}}\left(\mathscr{M}_{i}\right)\right)^{n k l}, \quad\left(\overline{\mathscr{L}_{c}}\right.\right.$ $\left.\left.\left(\mathscr{M}_{i}\right)\right)^{n k l}\right)$ denotes the largest value of the permutation from ith row and jth column of the collection $i \times j q-R O F S_{t} R N s$ $\left.\mathscr{L}_{c_{j}}\left(\mathscr{M}_{i}\right)=\underline{\left(\mathscr{L}_{c_{j}}\right.}\left(\mathscr{M}_{i}\right), \overline{\mathscr{L}_{c_{j}}}\left(\mathscr{M}_{i}\right)\right)$ and $n$ represents the balancing coefficient.
Example 6. Consider Tables 2, 4, and 5 of Examples 2 and 4, for the collection q-ROFS $t_{t}$ Ns $\mathscr{L}_{c}\left(\mathscr{M}_{i}\right)=\left(\mathscr{L}_{c_{j}}\left(\mathscr{M}_{i}\right)\right.$, $\left.\overline{\mathscr{L}_{c_{j}}}\left(\mathscr{M}_{i}\right)\right)$ with $k=(0.33,0.37,0.3)^{T}$ and $l=(0.42,0.58)^{T}$ is the weight vectors of experts $s_{i}$ and parameters $c_{j}$. Consider $t=(0.36,0.34,0.3)^{T}$ and $v=(0.55,0.45)^{T}$ as the associated weight vectors of experts $s_{i}$ and parameters $c_{j}$. Then, the aggregated result for $\mathscr{L}_{\delta c_{j}}^{*}\left(\mathscr{M}_{i}\right)$ is given as

$$
\begin{aligned}
& \mathrm{q}-\operatorname{ROFS}_{t} \operatorname{RHG}\left(\mathscr{L}_{c_{1}}\left(\mathscr{M}_{1}\right), \ldots, \mathscr{L}_{c_{n}}\left(\mathscr{M}_{m}\right)\right)=\left[\oplus_{j=1}^{2}\left\{\oplus_{i=1}^{3}\left(\underline{\mathscr{L}_{\delta c_{j}}}\left(\mathscr{M}_{i}\right)\right)^{t_{i}}\right\}^{v_{j}}, \oplus_{j=1}^{2}\left\{\oplus_{i=1}^{3}\left(\overline{\mathscr{L}}_{\delta c_{j}}\left(\mathscr{M}_{i}\right)\right)^{t_{i}}\right\}^{v_{j}}\right], \\
& \mathrm{q}-\operatorname{ROFS}_{t} \operatorname{RHG}\left(\mathscr{L}_{c_{1}}\left(\mathscr{M}_{1}\right), \ldots, \mathscr{L}_{c_{n}}\left(\mathscr{M}_{m}\right)\right)=((0.602629,0.250898),(0.479995,0.210973)) .
\end{aligned}
$$

From the analysis of Theorem 11, the properties of q-ROFS ${ }_{t}$ RHG operator are given below.

Theorem 11. Let $\mathscr{L}_{c_{j}}\left(\mathscr{M}_{i}\right)=\left(\mathscr{L}_{c_{j}}\left(\mathscr{M}_{i}\right), \overline{\mathscr{L}_{c_{j}}}\left(\mathscr{M}_{i}\right)\right)(i=$ $1,2, \ldots, m, j=1,2, \ldots, n)$ be the collection of $q-R^{2}{ } S_{t} R N s$. Let the weight vectors $k=\left(k_{1}, k_{2}, \ldots, k_{m}\right)^{T}$ and $l=\left(l_{1}, l_{2}, \ldots, l_{n}\right)^{T}$ of experts $s_{i}$ and parameters $c_{j}$ with $\sum_{i=1}^{m} t_{i}=1, \sum_{j=1}^{n} v_{j}=1$, and $0 \leq k_{i}, l_{j} \leq 1$. Consider the associated weight vectors $t=\left(t_{1}, t_{2}, \ldots, t_{m}\right)^{T}$ and $v=$ $\left(v_{1}, v_{2}, \ldots, v_{n}\right)^{T}$ of experts $s_{i}$ and parameters $c_{j}$ with $\sum_{i=1}^{m} t_{i}=1, \sum_{j=1}^{n} v_{j}=1$, and $0 \leq t_{i}, v_{j} \leq 1$, respectively. Then, $q-$ ROFS $_{t}$ RHG operator holds as follows:

(i) (Idempotency): if $\mathscr{L}_{\delta c_{j}}^{*}\left(\mathscr{M}_{i}\right)=\mathscr{P}_{c}(\mathcal{N}) \quad$ (for all $i=$ $1,2, \ldots$, m and $j=1,2, \ldots, n)$ where $\mathscr{P}_{c}(\mathscr{M})=\left(\mathscr{P}_{c}\right.$ $\left.(\mathscr{M}), \overline{\mathscr{P}}_{c}(\mathscr{M})\right)=((\underline{b}, \underline{d}),(\bar{b}, \bar{d}))$, then

$\mathrm{q}-\operatorname{ROFS}_{t} \operatorname{RHG}\left(\mathscr{L}_{c_{1}}\left(\mathscr{M}_{1}\right), \mathscr{L}_{c_{2}}\left(\mathscr{M}_{2}\right), \ldots, \mathscr{L}_{c_{n}}\left(\mathscr{M}_{m}\right)\right)=\mathscr{P}_{c}\left(\mathscr{M}_{)}\right)$

(ii) (Boundedness): let $\quad\left(\mathscr{L}_{\delta c_{j}}^{*}\left(\mathscr{M}_{i}\right)\right)^{-}=\left(\min _{j} \min _{i}\right.$ $\left.\mathscr{L}_{\delta c_{j}}^{*}\left(\mathscr{M}_{i}\right), \quad \max _{j} \max _{i} \overline{\mathscr{L}}_{\delta c_{j}}^{*}\left(\mathscr{M}_{i}\right)\right)$ and $\left(\mathscr{L}_{\delta c_{j}}^{*}\right.$
$\left.\left(\mathscr{M}_{i}\right)\right)^{+}=\left(\max _{j} \max _{i} \quad \underline{\mathscr{L}_{\delta c_{j}}^{*}}\left(\mathscr{M}_{i}\right), \min _{j} \min _{i} \overline{\mathscr{L}_{\delta c_{j}}^{*}}\right.$ $\left.\left(\mathscr{M}_{i}\right)\right)$. Then,

$$
\begin{aligned}
\left(\mathscr{L}_{\delta c_{j}}^{*}\left(\mathscr{M}_{i}\right)\right)^{-} & \leq \mathrm{q}-\operatorname{ROFS}_{t} \operatorname{RHG}\left(\mathscr{L}_{c_{1}}\left(\mathscr{M}_{1}\right), \mathscr{L}_{c_{2}}\left(\mathscr{M}_{2}\right), \ldots,\right. \\
\left.\mathscr{L}_{c_{n}}\left(\mathscr{M}_{m}\right)\right) & \leq\left(\mathscr{L}_{\delta c_{j}}^{*}\left(\mathscr{M}_{i}\right)\right)^{+}
\end{aligned}
$$

(iii) (Monotonicity): let $\mathscr{P}_{c_{j}}\left(\mathcal{N}_{i}\right)=\left(\mathscr{P}_{c_{j}}\left(\mathcal{N}_{i}\right), \overline{\mathscr{P}_{c_{j}}}\left(\mathcal{N}_{i}\right)\right)$ $(i=1,2, \ldots, m, j=1,2, \ldots, n) \overline{b e}$ another collection of $q-R O F S_{t} R N$ such that $\mathscr{P}_{c_{j}}\left(\mathscr{N}_{i}\right) \leq \mathscr{L}_{c_{j}}\left(\mathscr{M}_{i}\right)$ and $\overline{\mathscr{P}_{c_{j}}}\left(\mathscr{N}_{i}\right) \leq \overline{\mathscr{L}_{c_{j}}}\left(\mathscr{M}_{i}\right)$. Then,

$$
\begin{aligned}
\mathrm{q} & -\operatorname{ROFS}_{t} \operatorname{RHG}\left(\mathscr{P}_{c_{1}}\left(\mathscr{M}_{1}\right), \mathscr{P}_{c_{2}}\left(\mathscr{M}_{2}\right), \ldots, \mathscr{P}_{c_{n}}\left(\mathscr{M}_{m}\right)\right) \\
& \leq \mathrm{q}-\operatorname{ROFS}_{t} \operatorname{RHG}\left(\mathscr{L}_{c_{1}}\left(\mathscr{M}_{1}\right), \mathscr{L}_{c_{2}}\left(\mathscr{M}_{2}\right), \ldots, \mathscr{L}_{c_{n}}\left(\mathscr{M}_{m}\right)\right) .
\end{aligned}
$$

(iv) (Shift invariance): let $\mathscr{P}_{c}(\mathscr{N})=\left(\mathscr{P}_{c}(\mathcal{N}), \overline{\mathscr{P}_{c}}\right.$ $(\mathcal{N}))=((\underline{b}, \underline{d}),(\bar{b}, \bar{d}))$ be any other $q \overline{-R} O F S_{t} R N$. Then, 


$$
\begin{aligned}
\mathrm{q}- & \operatorname{ROFS} S_{t} \operatorname{RHG}\left(\mathscr{L}_{c_{1}}\left(\mathscr{M}_{1}\right) \oplus \mathscr{P}_{c}(\mathcal{N}), \mathscr{L}_{c_{2}}\left(\mathscr{M}_{2}\right)\right. \\
& \left.\oplus \mathscr{P}_{c}(\mathscr{N}), \ldots, \mathscr{L}_{c_{n}}\left(\mathscr{M}_{m}\right) \oplus \mathscr{P}_{c}(\mathcal{N})\right)=\mathrm{q}-\mathrm{ROFS}_{t} \mathrm{RHG} \\
& \left(\mathscr{L}_{c_{1}}\left(\mathscr{M}_{1}\right), \mathscr{L}_{c_{2}}\left(\mathscr{M}_{2}\right), \ldots, \mathscr{L}_{c_{n}}\left(\mathscr{M}_{m}\right)\right) \oplus \mathscr{P}_{c}(\mathcal{N}) .
\end{aligned}
$$

(v) (Homogeneity): for any real number $\lambda>0$,

$$
\begin{aligned}
\mathrm{q}- & \operatorname{ROFS} \operatorname{RHG}\left(\lambda \mathscr{L}_{c_{1}}\left(\mathscr{M}_{1}\right), \lambda \mathscr{L}_{c_{2}}\left(\mathscr{M}_{2}\right), \ldots,\right. \\
& \left.\lambda \mathscr{L}_{c_{n}}\left(\mathscr{M}_{m}\right)\right)=\lambda \mathrm{q}-\operatorname{ROFS}{ }_{t} \operatorname{RHG}\left(\mathscr{L}_{c_{1}}\left(\mathscr{M}_{1}\right), \mathscr{L}_{c_{2}}\right. \\
& \left.\left(\mathscr{M}_{2}\right), \ldots, \mathscr{L}_{c_{n}}\left(\mathscr{M}_{m}\right)\right) .
\end{aligned}
$$

Remark 7

(a) If $q=1$, then $q-\mathrm{ROFS}_{t} \mathrm{RHG}$ operator reduces to IFS $_{t}$ RHG operator

(b) If the value of rung $q=2$, then $\mathrm{q}-\mathrm{ROFS}_{t} \mathrm{RHG}$ operator reduces to $\mathrm{PFS}_{t} \mathrm{RHG}$ operator

(c) If the soft parameter $c_{1}$ is one (means $n=1$ ), then $\mathrm{q}-\mathrm{ROFS}_{t} \mathrm{RHG}$ operator reduces to q-ROFRHG operator

(d) If $k l=((1 / n),(1 / n), \ldots,(1 / n))^{T}$, then the proposed $\mathrm{q}-\mathrm{ROFS}_{t} \mathrm{RHG}$ operator reduces to $\mathrm{q}-\mathrm{ROFS}_{t} \mathrm{RWG}$ operator

(e) If $t v=((1 / n),(1 / n), \ldots,(1 / n))^{T}$, then the proposed q-ROFS RHG operator reduces to q-ROFS $_{t}$ ROWG operator

\section{MCDM Based on Soft Rough Aggregation Operator by Using q-ROF Information}

MCDM has a high potential and discipline process to improve and evaluate multiple conflicting criteria in all areas of decision-making. In this competitive environment, an enterprise needs a more accurate and more repaid response to change customer needs. So, MCDM has the ability to handle successfully the evaluation process of multiple contradictory criteria. For an intelligent decision, experts analyze each and every character of an alternative and, then, they take the decision. Further, we will present the model for MCDM and their basic steps of construction by utilizing the proposed aggregation operators under q-ROF soft rough information.

Suppose that $Y=\left\{s_{1}, s_{2}, s_{3}, \ldots, s_{p}\right\}$ be the initial set of various alternatives and $\mathscr{E}=\left\{c_{1}, c_{2}, c_{3}, \ldots, c_{n}\right\}$ be the set of $n$ parameters. Consider $\mathscr{Z}=\left\{z_{1}, z_{2}, z_{3}, \ldots, z_{m}\right\}$ as the set of $m$ professional experts of this area who present their assessment expertise for each alternative $s_{k}(k=1,2, \ldots, p)$ corresponding to $n$ parameters. Let the weight vectors $t=\left(t_{1}, t_{2}, \ldots, t_{m}\right)^{T}$ of experts $z_{i}$ and $v=\left(v_{1}, v_{2}, \ldots, v_{n}\right)^{T}$ of parameters $c_{j}$ with $\sum_{i=1}^{m} t_{i}=1, \sum_{j=1}^{n} v_{j}=1$, and $0 \leq t_{i}, v_{j} \leq 1$, respectively. The professional experts express their preference evaluation for alternative $s_{k}$ with respect to parameter $c_{j}$ in the form of q-ROFS ${ }_{t}$ RNs. The collective preference information given by the professionals is managed in q-ROFS ${ }_{t} \mathrm{R}$ decision matrix, which is $\mathbb{M}=\left[\mathscr{L}_{c_{j}}\left(\mathscr{M}_{i}\right)\right]_{n \times m}$ where $\mathscr{M} \subseteq \mathscr{E}$. Further, using the proposed models aggregate, the preferred choices of experts are to get the aggregated results $\mathscr{R}_{k}(k=1, \ldots, p)$ for each alternative $s_{k}$ against their parameter $c_{j}$. Finally, they utilize the score function on the aggregated results $\mathscr{R}_{k}=[(\beta, \underline{\vartheta}),(\bar{\beta}, \bar{\vartheta})]$ and rank all the results in a specific order to get the most desirable option. The stepwise decision algorithm for the investigated operators:

Step (i): the professional experts express their preference evaluation for alternative $s_{k}$ with respect to parameter $c_{j}$ in the form of $\mathrm{q}-\mathrm{ROFS}_{t} \mathrm{RNs}$. Then, they collect the preference information given by the professionals and manage them in $\mathrm{q}-\mathrm{ROFS}_{t} \mathrm{R}$ decision matrix, which is $\mathbb{M}=\left[\mathscr{L}_{c_{j}}\left(\mathscr{M}_{i}\right)\right]_{n \times m}$ where $\mathscr{M} \subseteq \mathscr{E}$.

Step (ii): apply the presented aggregation operators of each decision matrix $\mathbb{M}=\left[\mathscr{L}_{c_{j}}\left(\mathscr{M}_{i}\right)\right]_{n \times m}$ for each alternative $s_{k}(k=1,2, \ldots, p)$ against parameter $c_{j}$ to get the aggregated results $\mathscr{R}_{k}=[(\beta, \underline{\vartheta}),(\bar{\beta}, \bar{\vartheta})]$.

Step (iii): calculate the score value of aggregated results $\mathscr{R}_{k}=[(\beta, \underline{\vartheta}),(\bar{\beta}, \bar{\vartheta})]$ for each object $s_{k}$.

Step (iv): rank the score value of $\mathscr{R}_{k}$ in a specific order to get the optimum option of professional experts.

\section{Numerical Example}

In this section, we will initiate an illustrative example to prove the quality and excellency of the developed operators. Let the Higher Education Commission (HEC) in Pakistan plans to introduce a selection board of four high potential and professional professors $\mathscr{Z}=\left\{z_{1}, z_{2}, z_{3}, z_{4}\right\}$ from home and abroad to select the most desirable applicant. Out of many applicants, three applicants $Y=\left\{s_{1}, s_{2}, s_{3}\right\}$ were called for interviews. The interview mainly judges the applicants against some parameters $\mathscr{M}=\left\{c_{1}=\right.$ academic level, $c_{2}=$ development potential, $c_{3}=$ professional ethics, $c_{4}=$ research productivity $\subseteq \subseteq \mathscr{E}$. Let the weight vector $t=(0.3,0.28,0.24,0.18)^{T}$ for professional experts $z_{i}(i=1, \ldots, 4)$ and $v=(0.32,0.17,0.31,0.2)^{T}$ be the weight vectors for parameters $c_{j}(j=1,2,3)$, respectively. The professional experts express their preference evaluation for candidate $s_{k}$ with respect to parameter $c_{j}$ in the form of q-ROFS ${ }_{t}$ RNs. Finally, follow the following steps by utilizing the proposed models to select the most desirable and suitable applicant $s_{k}$.

\subsection{Aggregation Results Rendered by the $q-R O F S_{t} R W A$} Method

Step (i): the professional experts express their preference evaluation for alternative $s_{k}$ with respect to parameter $c_{j}$ in the form of $\mathrm{q}-\mathrm{ROFS}_{t} \mathrm{RNs}$. Then, they collect the preference information given by the professionals and manage them in q-ROFS $\mathrm{R}_{t}$ decision matrix, which is $\mathbb{M}=\left[\mathscr{L}_{c_{j}}\left(\mathscr{M}_{i}\right)\right]_{n \times m}$ where $\mathscr{M} \subseteq \mathscr{E}$ which is given in Tables 7-9.

Step (ii): apply the presented $\mathrm{q}-\mathrm{ROFS}_{t}$ RWA aggregation operators on each decision matrix 
TABLE 7: q-ROFS $S_{t} \mathrm{R}$ matrix for candidate $s_{1}$.

\begin{tabular}{ccccc}
\hline & $c_{1}$ & $c_{2}$ & $c_{3}$ & $c_{4}$ \\
\hline$z_{1}$ & {$[(0.7,0.2),(0.8,0.1)]$} & {$[(0.65,0.25),(0.3,0.6)]$} & {$[(0.82,0.18),(0.6,0.4)]$} & {$[(0.5,0.2),(0.4,0.1)]$} \\
$z_{2}$ & {$[(0.6,0.1),(0.5,0.3)]$} & {$[(0.5,0.1),(0.7,0.15)]$} & {$[(0.3,0.2),(0.2,0.7)]$} & {$[(0.2,0.3),(0.6,0.2)]$} \\
$z_{3}$ & {$[(0.4,0.5),(0.6,0.2)]$} & {$[(0.75,0.2),(0.4,0.1)]$} & {$[(0.65,0.3),(0.7,0.25)]$} & {$[(0.5,0.4),(0.1,0.5)]$} \\
$z_{4}$ & {$[(0.5,0.3),(0.3,0.7)]$} & {$[(0.6,0.4),(0.9,0.1)]$} & {$[(0.78,0.22),(0.45,0.4)]$} & {$[(0.8,0.1),(0.3,0.1)]$} \\
\hline
\end{tabular}

TABLE 8: q-ROFS $S_{t} \mathrm{R}$ matrix for candidate $s_{2}$.

\begin{tabular}{ccccc}
\hline & $c_{1}$ & $c_{2}$ & $c_{3}$ & $c_{4}$ \\
\hline$z_{1}$ & {$[(0.6,0.3),(0.9,0.1)]$} & {$[(0.2,0.4),(0.6,0.1)]$} & {$[(0.5,0.2),(0.9,0.1)]$} & {$[(0.6,0.2),(0.7,0.2)]$} \\
$z_{2}$ & {$[(0.4,0.25),(0.3,0.5)]$} & {$[(0.5,0.15),(0.7,0.3)]$} & {$[(0.77,0.1),(0.6,0.35)]$} & {$[(0.4,0.3),(0.5,0.1)]$} \\
$z_{3}$ & {$[(0.3,0.6),(0.65,0.25)]$} & {$[(0.66,0.2),(0.8,0.17)]$} & {$[(0.8,0.15),(0.55,0.2)]$} & {$[(0.7,0.1),(0.3,0.6)]$} \\
$z_{4}$ & {$[(0.5,0.15),(0.55,0.2)]$} & {$[(0.8,0.1),(0.4,0.5)]$} & {$[(0.62,0.3),(0.9,0.1)]$} & {$[(0.2,0.4),(0.5,0.3)]$} \\
\hline
\end{tabular}

TABLE 9: q-ROFS $S_{t} \mathrm{R}$ matrix for candidate $s_{3}$.

\begin{tabular}{ccccc}
\hline & $c_{1}$ & $c_{2}$ & $c_{3}$ & $c_{4}$ \\
\hline$z_{1}$ & {$[(0.8,0.13),(0.8,0.1)]$} & {$[(0.5,0.2),(0.6,0.1)]$} & {$[(0.4,0.1),(0.7,0.2)]$} & {$[(0.7,0.1),(0.6,0.3)]$} \\
$z_{2}$ & {$[(0.5,0.16),(0.4,0.2)]$} & {$[(0.8,0.12),(0.3,0.4)]$} & {$[(0.6,0.2),(0.4,0.3)]$} & {$[(0.5,0.2),(0.4,0.1)]$} \\
$z_{3}$ & {$[(0.4,0.5),(0.7,0.3)]$} & {$[(0.5,0.4),(0.5,0.12)]$} & {$[(0.2,0.4),(0.8,0.14)]$} & {$[(0.3,0.4),(0.2,0.5)]$} \\
$z_{4}$ & {$[(0.3,0.2),(0.5,0.15)]$} & {$[(0.6,0.25),(0.7,0.3)]$} & {$[(0.8,0.18),(0.75,0.1)]$} & {$[(0.6,0.2),(0.8,0.1)]$} \\
\hline
\end{tabular}

$\mathbb{M}=\left[\mathscr{L}_{c_{j}}\left(\mathscr{M}_{i}\right)\right]_{n \times m}$ for each alternative $s_{k}(k=1,2,3)$ against parameter $c_{j}$ to get the aggregated results $\mathscr{R}_{k}=[(\beta, \underline{\vartheta}),(\bar{\beta}, \bar{\vartheta})] ;$ that is,

$$
\begin{aligned}
& \mathscr{R}_{1}=[(0.640506,0.218382),(0.600249,0.254431)], \\
& \mathscr{R}_{2}=[(0.607809,0.217229),(0.730645,0.202415)], \\
& \mathscr{R}_{3}=[(0.606198,0.198551),(0.649417,0.183108)] .
\end{aligned}
$$

Step (iii): calculate the score value of aggregated results $\mathscr{R}_{k}=[(\beta, \underline{\vartheta}),(\bar{\beta}, \bar{\vartheta})]$ for each object $s_{k}$; that is,

$$
\begin{aligned}
& \operatorname{SC}\left(\mathscr{R}_{1}\right)=0.226074, \\
& \operatorname{SC}\left(\mathscr{R}_{2}\right)=0.298025, \\
& \operatorname{SC}\left(\mathscr{R}_{3}\right)=0.241341 .
\end{aligned}
$$

Step (iv): rank the score value of $\mathscr{R}_{k}$ in a specific order to get the optimum option of professional experts; that is,

$$
\operatorname{SC}\left(\mathscr{R}_{2}\right)>\operatorname{SC}\left(\mathscr{R}_{3}\right)>\operatorname{SC}\left(\mathscr{R}_{1}\right) \text {. }
$$

Therefore, from the above analysis, it is observed that $s_{2}$ is a more suitable and desirable candidate against the given position.

7.2. Aggregation Results Rendered by the $q-R O F S_{t} R W G$ Method

Step (i): similar to above.

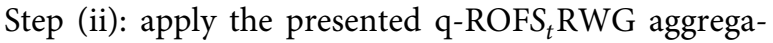
tion operators on each decision matrix
$\mathbb{M}=\left[\mathscr{L}_{c_{j}}\left(\mathscr{M}_{i}\right)\right]_{n \times m}$ for each alternative $s_{k}(k=1,2,3)$ against parameter $c_{j}$ to get the aggregated results $\mathscr{R}_{k}=[(\beta, \underline{\vartheta}),(\bar{\beta}, \bar{\vartheta})]$; that is,

$$
\begin{aligned}
& \mathscr{R}_{1}=[(0.540892,0.29117),(0.446263,0.448329)], \\
& \mathscr{R}_{2}=[(0.50166,0.321735),(0.598613,0.331553)], \\
& \mathscr{R}_{3}=[(0.491017,0.291113),(0.545527,0.264822)] .
\end{aligned}
$$

Step (iii): calculate the score value of the proposed q-ROFS ${ }_{t} \mathrm{RWG}$ aggregated results $\mathscr{R}_{k}=[(\beta, \underline{\vartheta}),(\bar{\beta}, \bar{\vartheta})]$ for each object $s_{k}$; that is,

$$
\begin{aligned}
& \operatorname{SC}\left(\mathscr{R}_{1}\right)=0.06616, \\
& \operatorname{SC}\left(\mathscr{R}_{2}\right)=0.135502, \\
& \operatorname{SC}\left(\mathscr{R}_{3}\right)=0.118744 .
\end{aligned}
$$

Step (iv): rank the score value of $\mathscr{R}_{k}$ in a specific order to get the optimum option of professional experts; that is,

$$
\operatorname{SC}\left(\mathscr{R}_{2}\right)>\operatorname{SC}\left(\mathscr{R}_{3}\right)>\operatorname{SC}\left(\mathscr{R}_{1}\right)
$$

Therefore, from the above analysis, it is observed that $s_{2}$ is a more suitable and desirable candidate against the given position.

7.3. Aggregation Results Rendered by the $q-\mathrm{ROFS}_{t} \mathrm{ROWA}$ Method

Step (i): similar to above. 
Step (ii):

$$
\begin{aligned}
& \mathscr{R}_{1}=[(0.657933,0.217542),(0.621125,0.234448)], \\
& \mathscr{R}_{2}=[(0.612743,0.216413),(0.744755,0.193923)], \\
& \mathscr{R}_{3}=[(0.621268,0.203392),(0.677814,0.180796)] .
\end{aligned}
$$

Step (iii): $\operatorname{SC}\left(\mathscr{R}_{1}\right)=0.250625, \operatorname{SC}\left(\mathscr{R}_{2}\right)=0.312857$, $\operatorname{SC}\left(\mathscr{R}_{3}\right)=0.26844$.

Step (iv): $\operatorname{SC}\left(\mathscr{R}_{2}\right)>\operatorname{SC}\left(\mathscr{R}_{3}\right)>\operatorname{SC}\left(\mathscr{R}_{1}\right)$.

Therefore, from the above analysis, it is observed that $s_{2}$ is a more suitable and desirable candidate against the given position.

\subsection{Aggregation Results Rendered by the $q-R O F S_{t} R O W G$ Method}

Step (i): similar to above.

Step (ii):

$$
\begin{aligned}
& \mathscr{R}_{1}=[(0.562833,0.293072),(0.473363,0.418793)], \\
& \mathscr{R}_{2}=[(0.517595,0.319441),(0.614971,0.321818)], \\
& \mathscr{R}_{3}=[(0.49826,0.296096),(0.583543,0.259781)] .
\end{aligned}
$$

Step (iii): $\operatorname{SC}\left(\mathscr{R}_{1}\right)=0.09287, \operatorname{SC}\left(\mathscr{R}_{2}\right)=0.152658$, $\operatorname{SC}\left(\mathscr{R}_{3}\right)=0.139459$.

Step (iv): $\operatorname{SC}\left(\mathscr{R}_{2}\right)>\operatorname{SC}\left(\mathscr{R}_{3}\right)>\operatorname{SC}\left(\mathscr{R}_{1}\right) \Rightarrow$

Therefore, from the above analysis, it is observed that $s_{2}$ is a more suitable and desirable candidate against the given position.

\subsection{Aggregation Results Rendered by the $q-$ ROFS $_{t} R H A$} Method

Step (i): similar to above.

Step (ii): apply the presented q-ROFS ${ }_{t}$ RHA aggregation operators on each decision matrix $\mathbb{M}=\left[\mathscr{L}_{\delta c_{j}}^{*}\left(\mathscr{M}_{i}\right)\right]_{n \times m}$ for each alternative $s_{k}(k=1,2,3)$ against parameter $c_{j}$ to get the aggregated results $\mathscr{R}_{k}=\left[\left(\underline{\beta_{\delta}^{*}}, \underline{\vartheta_{\delta}^{*}}\right),\left(\overline{\beta_{\delta}^{*}}, \overline{\vartheta_{\delta}^{*}}\right)\right]$, with $k=(0.25,0.29,0.3,0.16)^{T}$ and $l=(0.27,0.23$, $0.32,0.18)^{T}$ being the weight vectors of experts $s_{i}$ and parameters $c_{j}$. Then, the aggregated result for $\mathscr{L}_{\delta c_{j}}^{*}\left(\mathscr{M}_{i}\right)$ is given as

$$
\begin{aligned}
& \mathscr{R}_{1}=[(0.432025,0.669215),(0.408108,0.691652)], \\
& \mathscr{R}_{2}=[(0.417457,0.662671),(0.503394,0.6509)], \\
& \mathscr{R}_{3}=[(0.392067,0.655437),(0.442525,0.635251)] .
\end{aligned}
$$

Step (iii): $\operatorname{SC}\left(\mathscr{R}_{1}\right)=-0.24099, \operatorname{SC}\left(\mathscr{R}_{2}\right)=-0.18323$, $\operatorname{SC}\left(\mathscr{R}_{3}\right)=-0.1955$.

$$
\text { Step (iv): } \operatorname{SC}\left(\mathscr{R}_{2}\right)>\operatorname{SC}\left(\mathscr{R}_{3}\right)>\operatorname{SC}\left(\mathscr{R}_{1}\right) \text {. }
$$

Therefore, from the above analysis, it is observed that $s_{2}$ is a more suitable and desirable candidate against the given position.

7.6. Aggregation Results Rendered by the $q$-ROFS $\mathrm{RHG}_{t}$ Method

Step (i): similar to above.

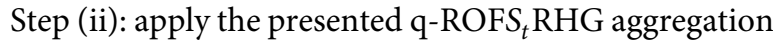
operators on each decision matrix $\mathbb{M}=\left[\mathscr{L}_{\delta c_{j}}^{*}\left(\mathscr{M}_{i}\right)\right]_{n \times m}$ for each alternative $s_{k}(k=1,2,3)$ against parameter $c_{j}$ to get the aggregated results $\mathscr{R}_{k}=\left[\left(\beta_{\delta}^{*}, \vartheta_{\delta}^{*}\right),\left(\overline{\beta_{\delta}^{*}}, \overline{\vartheta_{\delta}^{*}}\right)\right]$, with

$k=(0.25,0.29,0.3,0.16)^{T}$ and $l=$

$(0.27,0.23,0.32,0.18)^{T}$ being the weight vectors of experts $s_{i}$ and parameters $c_{j}$. Then, the aggregated result for $\mathscr{L}_{\delta c_{j}}^{*}\left(\mathscr{M}_{i}\right)$ is given as

$$
\begin{aligned}
& \mathscr{R}_{1}=[(0.365745,0.709626),(0.308693,0.751049)], \\
& \mathscr{R}_{2}=[(0.332518,0.713922),(0.407739,0.706165)], \\
& \mathscr{R}_{3}=[(0.318639,0.693983),(0.36813,0.673448)] .
\end{aligned}
$$

$$
\begin{aligned}
& \text { Step } \quad \text { (iii): } \quad \operatorname{SC}\left(\mathscr{R}_{1}\right)=-0.35133, \operatorname{SC}\left(\mathscr{R}_{2}\right)=- \\
& 0.30573, \operatorname{SC}\left(\mathscr{R}_{3}\right)=-0.27871 .
\end{aligned}
$$

Step (iv): $\operatorname{SC}\left(\mathscr{R}_{3}\right)>\operatorname{SC}\left(\mathscr{R}_{2}\right)>\operatorname{SC}\left(\mathscr{R}_{1}\right)$.

Therefore, from the above analysis, it is observed that $s_{3}$ is a more suitable and desirable candidate against the given position. The higher the score value, the more optimist that value and the smaller the score value, the more pessimist that value. From the ranking results of the above proposed operators, it is clear that averaging operators' results are more optimist than geometric operators.

7.7. Comparative Study. To present the applicability and efficiency of the developed approach with some other existing methods based on IFS, PFS, and q-ROFS methods by using the same illustrative example. A comparative study has been made based on different aggregation operators (see $[4,5,23,27,28,54,60,61])$. For this purpose, different parameters of the above numerical example are aggregated by utilizing the proposed aggregation operators having weight vectors $v=(0.32,0.17,0.31,0.2)^{T}$, and their collective aggregated decision matrix for each candidate $s_{i}(i=1,2,3)$ is given in Table 10. Now, by using the information of the evaluated matrix, a comparative study of the investigated models with some existing aggregation operators is presented in Table 11. From Table 11, it is observed that the methods presented in $[4,5,23,27,28,54,60,61]$ are only capable of solving the $\mathrm{q}$-ROFV of the form $(\beta, \vartheta)$ but are not capable of solving the $\mathrm{q}-\mathrm{ROFS}_{t} \mathrm{RV}$ of the form $[(\beta, \underline{\vartheta}),(\bar{\beta}, \bar{\vartheta})]$. Thus, from the existing methods, it is clear that these existing methods have a lake of rough information and they are not capable of solving and ranking the given illustrative example. Therefore, from this 
TABle 10: Aggregated matrix for candidate.

\begin{tabular}{llcl}
\hline & $s_{1}$ & $s_{2}$ & $s_{3}$ \\
\hline$z_{1}$ & {$[(0.719,0.201),(0.651,0.208)]$} & {$[(0.538,0.256),(0.849,0.115)]$} & {$[(0.670,0.122),(0.713,0.154)]$} \\
$z_{2}$ & {$[(0.471,0.154),(0.534,0.320)]$} & {$[(0.602,0.179),(0.550,0.298)]$} & {$[(0.616,0.171),(0.387,0.222)]$} \\
$z_{3}$ & {$[(0.598,0.350),(0.582,0.229)]$} & {$[(0.678,0.226),(0.629,0.260)]$} & {$[(0.370,0.430),(0.682,0.225)]$} \\
$z_{4}$ & {$[(0.703,0.230),(0.616,0.287)]$} & {$[(0.605,0.211),(0.733,0.205)]$} & {$[(0.650,0.201),(0.703,0.137)]$} \\
\hline
\end{tabular}

TABLE 11: Comparative analysis of different methods.

\begin{tabular}{|c|c|c|}
\hline \multirow{2}{*}{ Methods } & Score values & \multirow{2}{*}{ Ranking } \\
\hline & $\begin{array}{lll}\mathscr{R}_{1} & \mathscr{R}_{2} \quad \mathscr{R}_{3}\end{array}$ & \\
\hline IFWA [4] & Unapproachable & $\times$ \\
\hline $\mathrm{IFS}_{t} \mathrm{WA}[54]$ & Unapproachable & $\times$ \\
\hline $\mathrm{PFS}_{t} \mathrm{WA}[60]$ & Unapproachable & $x$ \\
\hline q-ROFWA [23] & Unapproachable & $x$ \\
\hline q-ROFHOWAGA [61] & Unapproachable & $\times$ \\
\hline $\mathrm{q}-\mathrm{ROFS}_{t} \mathrm{WA}[60]$ & Unapproachable & $x$ \\
\hline Cq-ROFWA [27] & Unapproachable & $\times$ \\
\hline q-RONFWA [28] & Unapproachable & $x$ \\
\hline \multicolumn{3}{|l|}{ q-ROFS ${ }_{t}$ RWA (proposed) } \\
\hline \multicolumn{2}{|c|}{$0.226074,0.298025,0.241341$} & $\mathscr{R}_{2} \geqslant \mathscr{R}_{3} \geqslant \mathscr{R}_{1}$ \\
\hline $\begin{array}{r}\text { q-ROFS } S_{t} \text { ROWA (proposed) } \\
0.250625,0.312857\end{array}$ & 6844 & $\mathscr{R}_{2} \geqslant \mathscr{R}_{3} \geqslant \mathscr{R}_{1}$ \\
\hline q-ROFS ${ }_{t}$ RHA (proposed) & $\begin{array}{c}-0.24099,- \\
0.18323,- \\
0.1955\end{array}$ & $\mathscr{R}_{2} \geqslant \mathscr{R}_{3} \geqslant \mathscr{R}_{1}$ \\
\hline IFWG [5] & Unapproachable & $x$ \\
\hline $\mathrm{IFS}_{t} \mathrm{WG}[54]$ & Unapproachable & $x$ \\
\hline q-ROFWG [23] & Unapproachable & $x$ \\
\hline Cq-ROFWG [27] & Unapproachable & $x$ \\
\hline q-RONFWG [28] & Unapproachable & $x$ \\
\hline \multicolumn{3}{|l|}{ 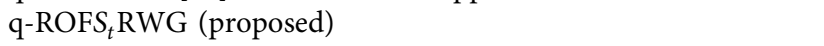 } \\
\hline \multicolumn{3}{|l|}{ 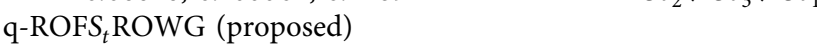 } \\
\hline \multicolumn{2}{|c|}{$0.09287,0.152658,0.139459$} & $\mathscr{R}_{2} \geqslant \mathscr{R}_{3} \geqslant \mathscr{R}_{1}$ \\
\hline $\mathrm{q}-\mathrm{ROFS}_{t}$ RHG (proposed) & $\begin{array}{c}-0.35133,- \\
0.30573,- \\
0.27871\end{array}$ & $\mathscr{R}_{3} \geqslant \mathscr{R}_{2} \geqslant \mathscr{R}_{1}$ \\
\hline
\end{tabular}

analysis, it is clear that the developed methods are more superior and capable than the existing methods.

\section{Conclusion}

MCDM has a high potential and discipline process to improve and evaluate multiple conflicting criteria in all areas of decision-making. For an intelligent decision, the experts analyze each and every character of an alternative and then they take the decision. For an intelligent and successful decision, the experts require a careful preparation and analysis of each and every character for an alternative and then they can take a good decision if they are armed with all the data and information that they need. The dominant notions of fuzzy sets, $S_{t} S s$, and rough sets generalized the classical set theory to cope with uncertain information. Molodtsov investigated the pioneer notion of $S_{t} S$ which is free from the inherent complexity which the contemporary theories faced. It is observed that $S_{t} S$ has too close relation with fuzzy sets and rough sets. The $S_{t} S$ theory is an effective mathematical tool for handling the uncertain, ambiguous, and imprecise data. The aim of our work is to investigate the hybrid concept of $S_{t} S$ and rough set with the notion of $\mathrm{q}$-ROFS to obtain the new notion of $\mathrm{q}-\mathrm{ROFS}_{t} \mathrm{RS}$. In addition, some averaging aggregation operators such as

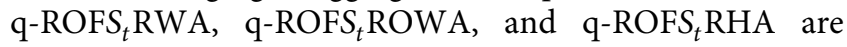
presented. Then, important properties of these investigated averaging operators are given in detail. Moreover, we investigated the geometric aggregation operators such as q-ROFS RWG, q-ROFS $_{t}$ ROWG, and q-ROFS RHG $^{\text {R }}$ and proposed the basic desirable characteristics of investigated geometric operators. The technique for MCDM and stepwise algorithm for decision-making by utilizing the proposed approaches are demonstrated clearly. Finally, a numerical example for the developed approach is presented and a comparative study of the investigated models with some existing methods is brought to light in detail which shows that the proposed models are more effective and superior than existing approaches.

8.1. Future Work. In the future, we intend to further discuss the following topics:

(i) The investigation of q-ROF-entropy of soft rough sets

(ii) The investigation of the picture and spherical fuzzy information by using soft rough sets

(iii) Applying other decision-making methodology based q-ROFS ${ }_{t}$ RS to solve the MCDM problem

(iv) The discussions of other applied methods in information systems

\section{Abbreviations}

IFS:

PFS:

q-ROFS:

$S_{t} \mathrm{~S}$ :

q-ROFS RS: $^{-}$

q-ROFS ${ }_{t}$ RWA:

q-

$\mathrm{ROFS}_{t}$ ROWA:

q-ROFS RHA: $^{-}$

q-ROFS RWG: $^{-}$

q-

$\mathrm{ROFS}_{t}$ ROWG:

q-ROFS RHG: $^{2}$

MCDM:

MemG:

NMemG:
Intuitionistic fuzzy set Pythagorean fuzzy set q-Rung orthopair fuzzy set Soft set q-ROF soft rough set q-ROF soft rough weighted averaging q-ROF soft rough ordered weighted averaging q-ROF soft rough hybrid averaging q-ROF soft rough weighted geometric q-ROF soft rough ordered weighted geometric q-ROF soft rough hybrid geometric Multicriteria decision-making Membership grade Nonmembership grade 
IFWA: IF weighted averaging

IFOWA: IF ordered weighted averaging

IFHA: IF hybrid averaging

IFWG: $\quad$ IF weighted geometric

IFOWG: IF ordered weighted geometric

IFHG: IF hybrid geometric

q-ROFWA: q-ROF weighted averaging

q-ROFWG: q-ROF weighted geometric

$\mathrm{IFS}_{t} \mathrm{~S}$ :

IFS,WA:

IFS, WG:

q-ROFS $S_{t}$ :

q-ROFS $S_{t}$ WA: q-ROF soft weighted averaging

q-ROFS $t$ OWA: q-ROF soft ordered weighted averaging

q-ROFS ${ }_{t} \mathrm{HA}: \quad$ q-ROF soft hybrid averaging

$\mathrm{q}-\mathrm{ROFS}_{t} \mathrm{~N}$ : $\quad \mathrm{q}$-ROF soft number

q-ROFS ${ }_{t} \mathrm{RN}: \quad \mathrm{q}-\mathrm{ROF}$ soft rough number.

\section{Data Availability}

The data used in this article are artificial and hypothetical, and anyone can use these data before prior permission by just citing this article.

\section{Conflicts of Interest}

The authors declare that they have no conflicts of interest.

\section{Acknowledgments}

This work was supported by Major Humanities and Social Sciences Research Projects in Zhejiang Universities (no. 2018QN058).

\section{References}

[1] L. A. Zadeh, "Fuzzy sets," Information and Control, vol. 8, no. 3, pp. 338-353, 1965.

[2] K. T. Atanassov, "Intuitionistic fuzzy sets," Fuzzy Sets Systems, vol. 20, no. 1, pp. 87-96, 1986.

[3] M. I. Ali, F. Feng, T. Mahmood, I. Mahmood, and H. Faizan, "A graphical method for ranking Atanassov's intuitionistic fuzzy values using the uncertainty index and entropy," International Journal of Intelligent Systems, vol. 34, no. 10, pp. 2692-2712, 2019.

[4] Z. S. Xu, "Intuitionistic fuzzy aggregation operators," IEEE Transection and Fuzzy Systems, vol. 15, pp. 1179-1187, 2007.

[5] Z. Xu and R. R. Yager, "Some geometric aggregation operators based on intuitionistic fuzzy sets," International Journal of General Systems, vol. 35, no. 4, pp. 417-433, 2006.

[6] H. Zhao, Z. Xu, M. Ni, and S. Liu, "Generalized aggregation operators for intuitionistic fuzzy sets," International Journal of Intelligent Systems, vol. 25, no. 1, pp. 1-30, 2010.

[7] W. Wang and X. Liu, "Intuitionistic fuzzy geometric aggregation operators based on Einstein operations," International Journal of Intelligent Systems, vol. 26, no. 11, pp. 1049-1075, 2011.

[8] W. Wang and X. Liu, "Intuitionistic fuzzy information aggregation using Einstein operations," IEEE Transactions on Fuzzy Systems, vol. 20, no. 5, pp. 923-938, 2012.
[9] S. Zeng, S.-M. Chen, and L.-W. Kuo, "Multiattribute decision making based on novel score function of intuitionistic fuzzy values and modified VIKOR method," Information Sciences, vol. 488, pp. 76-92, 2019.

[10] R. R. Yager, "Pythagorean fuzzy subsets," in Proceedings of the Annual Meeting of Joint IFSA World Congress and NAFIPS, vol. 24, no. 28, pp. 57-61, Edmonton, Canada, 2013.

[11] R. R. Yager, "Pythagorean membership grades in multicriteria decision making," IEEE Transactions on Fuzzy Systems, vol. 22, no. 4, pp. 958-965, 2014.

[12] X. Peng and Y. Yang, "Some results for Pythagorean fuzzy sets," International Journal of Intelligent Systems, vol. 30, no. 11, pp. 1133-1160, 2015.

[13] X. Peng and Y. Yang, "Pythagorean fuzzy choquet integral based MABAC method for multiple attribute group decision making," International Journal of Intelligent Systems, vol. 31, no. 10, pp. 989-1020, 2016.

[14] H. Garg, "A new generalized Pythagorean fuzzy information aggregation using Einstein operations and its application to decision making," International Journal of Intelligent Systems, vol. 31, no. 9, pp. 886-920, 2016.

[15] H. Garg, "Generalized Pythagorean fuzzy geometric aggregation operators using Einstein $t$-norm and $t$-conorm for multicriteria decision-making process," International Journal of Intelligent Systems, vol. 32, no. 6, pp. 597-630, 2017.

[16] H. Garg, "Confidence levels based Pythagorean fuzzy aggregation operators and its application to decision-making process," Computational and Mathematical Organization Theory, vol. 23, no. 4, pp. 546-571, 2017.

[17] G. Wei and M. Lu, "Pythagorean fuzzy power aggregation operators in multiple attribute decision making," International Journal of Intelligent Systems, vol. 33, no. 1, pp. 169-186, 2018.

[18] G. Wei, "Pythagorean fuzzy interaction aggregation operators and their application to multiple attribute decision making," Journal of Intelligent \& Fuzzy Systems, vol. 33, no. 4, pp. 2119-2132, 2017.

[19] S.-J. Wu and G.-W. Wei, "Pythagorean fuzzy Hamacher aggregation operators and their application to multiple attribute decision making," International Journal of KnowledgeBased and Intelligent Engineering Systems, vol. 21, no. 3, pp. 189-201, 2017.

[20] R. R. Yager, "Generalized orthopair fuzzy sets," IEEE Transactions on Fuzzy Systems, vol. 25, no. 5, pp. 1222-1230, 2017.

[21] R. R. Yager and N. Alajlan, "Approximate reasoning with generalized orthopair fuzzy sets," Information Fusion, vol. 38, pp. 65-73, 2017.

[22] M. I. Ali, "Another view on q-rung orthopair fuzzy sets," International Journal of Intelligent Systems, vol. 33, pp. 2139-2153, 2019.

[23] P. Liu and P. Wang, "Some q-rung orthopair fuzzy aggregation operators and their applications to multiple-attribute decision making," International Journal of Intelligent Systems, vol. 33, no. 2, pp. 259-280, 2018.

[24] P. Liu and J. Liu, "Some q-rung orthopai fuzzy Bonferroni mean operators and their application to multi-attribute group decision making," International Journal of Intelligent Systems, vol. 33, no. 2, pp. 315-347, 2018.

[25] C. Jana, G. Muhiuddin, and M. Pal, "Some Dombi aggregation of q-rung orthopair fuzzy numbers in multiple-attribute decision making," International Journal of Intelligent Systems, vol. 34, no. 12, pp. 3220-3240, 2019. 
[26] J. Wang, R. Zhang, X. Zhu, Z. Zhou, X. Shang, and W. Li, "Some q-rung orthopair fuzzy Muirhead means with their application to multi-attribute group decision making," Journal of Intelligent \& Fuzzy Systems, vol. 36, no. 2, pp. 1599-1614, 2019.

[27] B. P. Joshi and A. Gegov, "Confidence levels q-rung orthopair fuzzy aggregation operators and its applications to MCDM problems," International Journal of Intelligent Systems, vol. 35, no. 1, pp. 125-149, 2020.

[28] Z. Yang, X. Li, Z. Cao, and J. Li, "q-rung orthopair normal fuzzy aggregation operators and their application in multiattribute decision-making," Mathematics, vol. 7, no. 12, p. 1142, 2019.

[29] A. Hussain, M. I. Ali, and T. Mahmood, "Hesitant q-rung orthopair fuzzy aggregation operators with their applications in multi-criteria decision making," Iranian Journal of Fuzzy Systems, vol. 17, no. 3, pp. 117-134, 2020.

[30] A. Hussain, M. I. Ali, T. Mahmood, and M. Munir, "Groupbased generalized q-rung orthopair average aggregation operators and their applications in multi-criteria decision making," Complex and Intelligent Systems, 2020.

[31] Z. a. Pawlak, "Rough sets," International Journal of Computer \& Information Sciences, vol. 11, no. 5, pp. 341-356, 1982.

[32] M. I. Ali, B. Davvaz, and M. Shabir, "Some properties of generalized rough sets," Information Sciences, vol. 224, pp. 170-179, 2013.

[33] D. Chen, C. Wang, and Q. Hu, "A new approach to attribute reduction of consistent and inconsistent covering decision systems with covering rough sets," Information Sciences, vol. 177, no. 17, pp. 3500-3518, 2007.

[34] T.-J. Li, Y. Leung, and W.-X. Zhang, "Generalized fuzzy rough approximation operators based on fuzzy coverings," International Journal of Approximate Reasoning, vol. 48, no. 3, pp. 836-856, 2008.

[35] M. I. Ali, T. Mahmood, and A. Hussain, "A study of generalized roughness in-fuzzy filters of ordered semigroups," Journal of Taibah University for Science, vol. 12, no. 2, pp. 163-172, 2018.

[36] T. Mahmood, M. I. Ali, and A. Hussain, "Generalized roughness in fuzzy filters and fuzzy ideals with thresholds in ordered semigroups," Computational and Applied Mathematics, vol. 37, no. 4, pp. 5013-5033, 2018.

[37] A. Hussain, M. I. Ali, and T. Mahmood, "Generalized roughness of q-fuzzy ideals in ordered semigroups," Journal of New Theory, vol. 26, pp. 32-53, 2019.

[38] W. Zhu, "Generalized rough sets based on relations," Information Sciences, vol. 177, no. 22, pp. 4997-5011, 2007.

[39] K. Chakrabarty, T. Gedeon, and L. Koczy, "Intuitionistic fuzzy rough set," in Proceedings of 4th Joint Conference on Information Sciences (JCIS), pp. 211-214, Durham, NC, USA, 1998.

[40] B. Huang, D.-k. Wei, H.-x. Li, and Y.-l. Zhuang, "Using a rough set model to extract rules in dominance-based intervalvalued intuitionistic fuzzy information systems," Information Sciences, vol. 221, pp. 215-229, 2013.

[41] S. P. Jena, S. K. Ghosh, and B. K. Tripathy, "Intuitionistic fuzzy rough sets," Notes Intuitionistic Fuzzy Sets, vol. 8, no. 1, pp. 1-18, 2002.

[42] S. K. Samanta and T. K. Mondal, "Intuitionistic fuzzy rough sets and rough intuitionistic fuzzy sets," Journal of Fuzzy Mathematics, vol. 9, no. 3, pp. 561-582, 2001.

[43] L. Zhou, W.-Z. Wu, and W.-X. Zhang, "On characterization of intuitionistic fuzzy rough sets based on intuitionistic fuzzy implicators," Information Sciences, vol. 179, no. 7, pp. 883-898, 2009.
[44] L. Zhou and W.-Z. Wu, "Characterization of rough set approximations in Atanassov intuitionistic fuzzy set theory," Computers \& Mathematics with Applications, vol. 62, no. 1, pp. 282-296, 2011.

[45] L. Zhou and W. Z. Wu, "On generalized intuitionistic fuzzy rough approximation operators," Information Sciences, vol. 178 , no. 11 , pp. $2448-2465,2008$.

[46] A. Hussain, T. Mahmood, and M. I. Ali, "Rough Pythagorean fuzzy ideals in semigroups," Computational and Applied Mathenatics, vol. 38, no. 2, p. 67, 2019.

[47] S. Zeng, "Pythagorean fuzzy multiattribute group decision making with probabilistic information and OWA approach," International Journal of Intelligent Systems, vol. 32, no. 11, pp. 1136-1150, 2017.

[48] A. Hussain, M. Irfan Ali, and T. Mahmood, "Covering based q-rung orthopair fuzzy rough set model hybrid with TOPSIS for multi-attribute decision making," Journal of Intelligent \& Fuzzy Systems, vol. 37, no. 1, pp. 981-993, 2019.

[49] D. Molodtsov, "Soft set theory-first results," Computers \& Mathematics with Applications, vol. 37, no. 4-5, pp. 19-31, 1999.

[50] P. K. Maji, R. Biswas, and A. R. Roy, "Fuzzy soft sets," Journal of Fuzzy Mathematics, vol. 9, pp. 589-602, 2001.

[51] P. K. Maji, R. Biswas, and A. R. Roy, "Intuitionistic fuzzy soft sets," Journal of Fuzzy Mathematics, vol. 9, pp. 677-692, 2001.

[52] M. I. Ali, F. Feng, X. Liu, W. K. Min, and M. Shabir, "On some new operations in soft set theory," Computers \& Mathematics with Applications, vol. 57, no. 9, pp. 1547-1553, 2009.

[53] M. Agarwal, K. K. Biswas, and M. Hanmandlu, "Generalized intuitionistic fuzzy soft sets with applications in decisionmaking," Applied Soft Computing, vol. 13, no. 8, pp. 3552-3566, 2013.

[54] R. Arora and H. Garg, "A robust aggregation operator for multi-criteria decision-making with intuitionistic fuzzy soft set environment," Scientia Iranica, vol. 25, no. 2, pp. 913-942, 2018.

[55] H. Garg and R. Arora, "Generalized intuitionistic fuzzy soft power aggregation operator based on $t$-norm and their application in multicriteria decision-making," International Journal of Intelligent Systems, vol. 34, no. 2, pp. 215-246, 2019.

[56] R. Arora, "Intuitionistic fuzzy soft aggregation operator based on Einstein norms and its applications in decision-making," in Proceedings of the 2018 International Conference on Intelligent Systems Design and Applications, Vellore, India, December 2018.

[57] F. Feng, H. Fujita, M. I. Ali, R. R. Yager, and X. Liu, “Another view on generalized intuitionistic fuzzy soft sets and related multi attribute decision making methods," IEEE Transactions on Fuzzy Systems, vol. 27, pp. 474-488, 2018.

[58] A. Hussain, M. I. Ali, and T. Mahmood, "Pythagorean fuzzy soft rough sets and their applications in decision-making," Journal of Taibah University for Science, vol. 14, no. 1, pp. 101-113, 2020.

[59] M. Riaz and M. R. Hashmi, "Soft rough Pythagorean m-polar fuzzy sets and Pythagorean m-polar fuzzy soft rough sets with application to decision-making," Computational and Applied Mathematics, vol. 39, no. 1, p. 16, 2020.

[60] A. Hussain, M. I. Ali, T. Mahmood, and M. Munir, "q-rung orthopair fuzzy soft average aggregation operators and their application in multicriteria decision-making," International Journal of Intelligent Systems, vol. 35, no. 4, pp. 571-599, 2020.

[61] M. Riaz, H. M. A. Farid, F. Karaaslan, and M. R. Hashmi, "Some q-rung orthopair fuzzy hybrid aggregation operators and TOPSIS method for multi-attribute decision-making," Journal of Intelligent and Fuzzy Systems, vol. 32, no. 2, pp. 259-280, 2020. 